TRANSACTIONS OF THE

AMERICAN MATHEMATICAL SOCIETY

Volume 365, Number 3, March 2013, Pages 1251-1276

S 0002-9947(2012)05831-4

Article electronically published on November 27, 2012

\title{
COMPACT AND WEAKLY COMPACT DISJOINTNESS PRESERVING OPERATORS ON SPACES OF DIFFERENTIABLE FUNCTIONS
}

\author{
DENNY H. LEUNG AND YA-SHU WANG
}

\begin{abstract}
A pair of functions defined on a set $X$ with values in a vector space $E$ is said to be disjoint if at least one of the functions takes the value 0 at every point in $X$. An operator acting between vector-valued function spaces is disjointness preserving if it maps disjoint functions to disjoint functions. We characterize compact and weakly compact disjointness preserving operators between spaces of Banach space-valued differentiable functions.
\end{abstract}

Let $X, Y$ be topological spaces and let $E, F$ be Banach spaces. Suppose that $A(X, E)$ and $A(Y, F)$ are subspaces of the spaces of continuous functions $C(X, E)$ and $C(Y, F)$ respectively. An operator $T: A(X, E) \rightarrow A(Y, F)$ is said to be disjointness preserving or separating if $T$ maps disjoint functions to disjoint functions. Here disjointness of two functions $f$ and $g$ in, say, $A(X, E)$ simply means that either $f(x)=0$ or $g(x)=0$ for all $x \in X$. In case $E=F=\mathbb{R}$, any algebraic homomorphism $T: C(X) \rightarrow C(Y)$ is disjointness preserving. The study of such homomorphisms is classical; see, for example, [8]. Disjointness preserving operators on spaces of continuous functions, whether scalar- or vector-valued, have also been investigated extensively; a by-no-means exhaustive list of references includes [1, 3, 5, 11. We also note that a complete characterization of disjointness preserving operators $T: C(X) \rightarrow C(Y)$ is obtained in [12. A similar result for operators $T: C_{0}(X) \rightarrow C_{0}(Y), X, Y$ locally compact, was obtained in [16, as well as characterizations of compactness and weak compactness of $T$. These results were extended to the vector-valued case in [13. In a parallel direction, many authors have studied algebraic homomorphisms between spaces of scalar-valued differentiable functions 4, 9, 10. In the vector-valued case, Araujo 2] gave a characterization of biseparating maps $T: C^{p}(X, E) \rightarrow C^{q}(Y, F), p, q<\infty$. (A map $T$ is biseparating if it is a bijection and both $T$ and $T^{-1}$ are disjointness preserving.)

In this paper, we undertake a thorough study of compact and weakly compact disjointness preserving operators $T: C^{p}(X, E) \rightarrow C^{q}(Y, F)$. Recall that a linear map $T$ between topological vector spaces is said to be compact, respectively, weakly compact if $T$ maps some open set onto a relatively compact, respectively, relatively weakly compact set. Our results can be compared in particular with those of [9, 10], which concern homomorphisms on spaces of scalar-valued differentiable

Received by the editors March 11, 2011.

2010 Mathematics Subject Classification. Primary 46E40, 46E50, 47B33, 47B38.

Key words and phrases. Disjointness preserving operators, spaces of vector-valued differentiable functions, compact and weakly compact operators.

The research of the first author was partially supported by AcRF project no. R-146-000-157112 .

(c) 2012 American Mathematical Society 
functions. (Note, however, that in [9], a (weakly) compact operator is defined to be one that maps bounded sets onto relatively (weakly) compact sets.) A major difference between the present situation and the case of homomorphisms treated in [9, 10, is that the operator $T$ is represented as a series rather than a single term (see Theorem 10). Because of the interference between terms, extraction of information on the various components of the representation (i.e., the terms $\Phi_{k}$ and $h$ in Theorem 10 becomes a much more delicate business.

We now briefly summarize the contents of the various sections of the paper. In $\S 1$, a variation of the Stone-Čech compactification procedure is introduced to obtain the support map of a disjointness preserving operator. In $\S 2$, based on a concentration argument (Proposition 9), a general representation theorem for continuous disjointness preserving operators between spaces of vector-valued differentiable functions is obtained (Theorem 10). The next three sections are devoted to a close study of the support map in various situations. This study, especially in the cases $p=q=\infty$ and $p=q<\infty$, requires careful analysis of the map $T$ utilizing the representation in Theorem 10. The result of the analysis is that the support map is locally constant unless $p>q$. In $\S 6$, the compactness or weak compactness of $T$ is taken into account. First, in Proposition 25, we embed $C^{q}(Y, F)$ linearly homeomorphically into a product of Banach spaces of (vector-valued) continuous functions. Then, using well-known characterizations of compactness and weak compactness in spaces of continuous functions, we reach the goals of characterizing compact and weakly compact disjointness preserving operators $T: C^{p}(X, E) \rightarrow C^{q}(Y, F)$ in all cases except where $p>q$. In the final section, it is shown that when $p>q$, not only is the support map $h$ not necessarily locally constant, it does not even have to be differentiable at all points. Nevertheless, we show that $h$ is $C^{q}$ on a dense open set and deduce characterizations of compact and weakly compact disjointness preserving operators.

The first author thanks Wee-Kee Tang for many discussions concerning the results of the paper.

\section{Compactification}

Let $X$ be a Hausdorff topological space and let $E$ be a Banach space. Suppose that $A(X)$ is a vector subspace of $C(X)$, the space of all continuous real-valued functions on $X$. Assume that $A(X)$ separates points from closed sets: If $x \in X$ and $P$ is a closed subset of $X$ not containing $x$, then there exists $\varphi \in A(X)$ so that $\varphi(x)=1$ and $\varphi(P) \subseteq\{0\}$. Let $A(X, E)$ be a vector subspace of $C(X, E)$, the space of $E$-valued continuous functions on $X$, that is $A(X)$-multiplicative: $\varphi f \in A(X, E)$ if $\varphi \in A(X)$ and $f \in A(X, E)$. The use of $A(X)$-compactification (defined below) in the study of separating maps is inspired by a similar construction in [1].

Proposition 1. Define $i: X \rightarrow \mathbb{R}^{A(X)}$ by $i(x)(\varphi)=\varphi(x)$. Then $i$ is a homeomorphic embedding.

Proof. If $x_{1} \neq x_{2}$, there exists $\varphi \in A(X)$ such that $\varphi\left(x_{1}\right) \neq \varphi\left(x_{2}\right)$. It follows that $i$ is one-to-one.

Since each $\varphi \in A(X)$ is continuous, $i$ is also continuous.

Suppose $x_{0} \in X$ and $U$ is an open neighborhood of $x_{0}$ in $X$. Set $P=X \backslash U$. There exists $\varphi \in A(X)$ such that $\varphi\left(x_{0}\right)=1$ and $\varphi(P) \subseteq\{0\}$. Now $V=\{a \in$ $\left.\mathbb{R}^{A(X)}: a(\varphi)>0\right\}$ is an open neighborhood of $i\left(x_{0}\right)$ in $\mathbb{R}^{A(X)}$. If $x \in X$ and 
$i(x) \in V$, then $\varphi(x)=i(x)(\varphi)>0$ and hence $x \notin P$. Thus $x \in U$. This establishes the continuity of $i^{-1}$ on $i(X)$.

Denote by $\mathbb{R}_{\infty}$ the 1 -point compactification of $\mathbb{R}$ and regard $\mathbb{R}^{A(X)}$ naturally as a subspace of $\mathbb{R}_{\infty}^{A(X)}$. We call the closure of $i(X)$ in $\mathbb{R}_{\infty}^{A(X)}$ the $A(X)$-compactification of $X$ and denote it by $\mathcal{A} X$. Note that $\mathcal{A} X$ is indeed compact Hausdorff and $X$ is homeomorphic with the dense subspace $i(X)$ in $\mathcal{A} X$.

Assume from here on that $A(X)$ has the following property $(*)$ :

For any $N \in \mathbb{N}$, any $\varphi_{1}, \ldots, \varphi_{N}$ in $A(X)$ and any $C^{\infty}$ function $\Phi: \mathbb{R}^{N} \rightarrow \mathbb{R}$ with $\sup _{a \in \mathbb{R}^{N}}\left|\partial^{\xi} \Phi(a)\right|<\infty$ for all $\xi$, the function

$$
\Phi\left(\varphi_{1}, \ldots, \varphi_{N}\right): x \mapsto \Phi\left(\varphi_{1}(x), \ldots, \varphi_{N}(x)\right) \text { belongs to } A(X) .
$$

We omit the simple proof of the next lemma.

Lemma 2. If $P^{\prime}$ and $Q^{\prime}$ are sets in $\mathbb{R}^{N}$ such that $d\left(P^{\prime}, Q^{\prime}\right)>0$, where $d$ is the usual Euclidean distance on $\mathbb{R}^{N}$, then there exists a $C^{\infty}$ function $\Phi: \mathbb{R}^{N} \rightarrow \mathbb{R}$ with $\sup _{a \in \mathbb{R}^{N}}\left|\partial^{\xi} \Phi(a)\right|<\infty$ for all $\xi$ such that $\Phi\left(P^{\prime}\right) \subseteq\{0\}$ and $\Phi\left(Q^{\prime}\right) \subseteq\{1\}$.

Proposition 3. Assume that $A(X)$ separates points from closed sets and has property (*). If $P$ and $Q$ are subsets of $X$ with $\overline{i(P)}^{\mathcal{A} X} \cap \overline{i(Q)}^{\mathcal{A} X}=\emptyset$, then there exists $\varphi \in A(X)$ so that $\varphi(P) \subseteq\{0\}$ and $\varphi(Q) \subseteq\{1\}$.

Proof. Let $P$ and $Q$ be as above. By compactness of $\overline{i(P)}^{\mathcal{A} X}$ and $\overline{i(Q)} \mathcal{A} X$, there are two finite collections $\mathcal{U}_{1}$ and $\mathcal{U}_{2}$ of basic open sets in $\mathbb{R}_{\infty}^{A(X)}$ so that $i(P) \subseteq \cup \mathcal{U}_{1}$, $i(Q) \subseteq \cup \mathcal{U}_{2}$ and $\bigcup \mathcal{U}_{1}, \cup \mathcal{U}_{2}$ have disjoint closures in $\mathbb{R}_{\infty}^{A(X)}$. Each basic open set $U$ in $\mathbb{R}_{\infty}^{A(X)}$ is of the form

$$
\left\{a \in \mathbb{R}_{\infty}^{A(X)}: a(\varphi) \in V_{\varphi}(U) \text { for all } \varphi \in S(U)\right\},
$$

where $S(U)$ is a finite subset of $A(X)$ and $V_{\varphi}(U)$ is open in $\mathbb{R}_{\infty}$ for all $\varphi \in S(U)$. If some $V_{\varphi}(U)$ contains $\infty$, replace it with $V_{\varphi}(U) \cap \mathbb{R}$. The modified collections of basic open sets still cover $i(P)$ and $i(Q)$ respectively. Let $S=\bigcup\left\{S(U): U \in \mathcal{U}_{1} \cup \mathcal{U}_{2}\right\}$. Then $S$ is a finite subset of $A(X)$. Denote by $P^{\prime}$ and $Q^{\prime}$ the respective projections of $i(P)$ and $i(Q)$ onto $\mathbb{R}^{S}$. We claim that $d\left(P^{\prime}, Q^{\prime}\right)>0$. If the claim is false, there are sequences $\left(p_{n}\right)$ and $\left(q_{n}\right)$ in $P$ and $Q$ respectively so that $d\left(\rho\left(i\left(p_{n}\right)\right), \rho\left(i\left(q_{n}\right)\right)\right) \rightarrow 0$, where $\rho$ is the projection from $\mathbb{R}^{A(X)}$ onto $\mathbb{R}^{S}$. This implies that $\left|\varphi\left(p_{n}\right)-\varphi\left(q_{n}\right)\right| \rightarrow 0$ for all $\varphi \in S$. It follows that there is an increasing sequence $\left(n_{k}\right)$ in $\mathbb{N}$ so that for all $\varphi \in S,\left(\varphi\left(p_{n_{k}}\right)\right)$ and $\left(\varphi\left(q_{n_{k}}\right)\right)$ both converge to the same $r_{\varphi}$ in $\mathbb{R}_{\infty}$. Define $r_{\varphi} \in \mathbb{R}_{\infty}$ arbitrarily for $\varphi \notin S$. Then $r=\left(r_{\varphi}\right)$ lies in the closure of both $\bigcup \mathcal{U}_{1}$ and $\bigcup \mathcal{U}_{2}$ in $\mathbb{R}_{\infty}^{A(X)}$, a contradiction. So the claim is verified.

Let $\Phi: \mathbb{R}^{S} \rightarrow \mathbb{R}$ be a function given by Lemma 2. By property $(*), \Phi\left((\varphi)_{\varphi \in S}\right) \in$ $A(X)$. If $x \in P,(\varphi(x))_{\varphi \in S}=\rho(i(x)) \in P^{\prime}$ and hence $\Phi\left((\varphi(x))_{\varphi \in S}\right)=0$. Similarly, $\Phi\left((\varphi(x))_{\varphi \in S}\right)=1$ for all $x \in Q$.

Let $Y$ be a Hausdorff topological space and let $F$ be a Banach space. Suppose that $A(Y, F)$ is a vector subspace of $C(Y, F)$. Two functions $f_{1}, f_{2} \in A(X, E)$ are said to be disjoint, denoted $f_{1} \perp f_{2}$, if for all $x \in X$, either $f_{1}(x)=0$ or $f_{2}(x)=0$. Disjointness for functions in $A(Y, F)$ is defined similarly. A map $T$ : $A(X, E) \rightarrow A(Y, F)$ is said to be separating or disjointness preserving if $f_{1} \perp f_{2}$ implies $T f_{1} \perp T f_{2}$. In the sequel, for $f \in A(X, E)$, let $C(f)=\{x \in X: f(x) \neq 0\}$ and let $\bar{C}(f)$ be the closure of $i(C(f))$ in $\mathcal{A} X$. 
Proposition 4. Let $T: A(X, E) \rightarrow A(Y, F)$ be a linear separating map. Then there is a continuous function $h: \tilde{Y} \rightarrow \mathcal{A X}$,

$$
\tilde{Y}=\{y \in Y: \text { there exists } f \in A(X, E) \text { with } T f(y) \neq 0\},
$$

so that if $f \in A(X, E)$ and $h(y) \notin \bar{C}(f)$, then $T f(y)=0$.

We will call $h$ the support map of the operator $T$. The proof of the proposition is decomposed into the next three lemmas. For any $y \in Y$, Let $\mathcal{O}_{y}$ be the family of all open subsets $U$ of $\mathcal{A} X$ so that there exists $f \in A(X, E)$ with $T f(y) \neq 0$ and $\bar{C}(f) \subseteq U$.

Lemma 5. $\mathcal{O}_{y}$ is closed under finite intersections.

Proof. Suppose that $U_{1}, U_{2} \in \mathcal{O}_{y}$ and $f_{1}, f_{2}$ are as in the definition. Choose open sets $V_{1}, V_{2}$ in $\mathcal{A} X$ so that

$$
\bar{C}\left(f_{1}\right) \cap \bar{C}\left(f_{2}\right) \subseteq V_{1} \subseteq{\overline{V_{1}}}^{\mathcal{A} X} \subseteq V_{2} \subseteq{\overline{V_{2}}}^{\mathcal{A} X} \subseteq U_{1} \cap U_{2}
$$

Let $P=i^{-1}\left(V_{1}\right)$ and $Q=i^{-1}\left(\mathcal{A} X \backslash{\overline{V_{2}}}^{\mathcal{A} X}\right)$. Then $\overline{i(P)}^{\mathcal{A} X} \subseteq{\overline{V_{1}}}^{\mathcal{A} X}$ and $\overline{i(Q)}^{\mathcal{A} X} \subseteq$ $\mathcal{A} X \backslash V_{2}$ and hence $\overline{i(P)} \mathcal{A} X \cap \bar{i}^{\mathcal{A} X} \mathcal{A}=\emptyset$. By Proposition 3 there exists $\varphi \in A(X)$ so that $\varphi(P) \subseteq\{0\}$ and $\varphi(Q) \subseteq\{1\}$. Then $\varphi f_{j} \in A(X, E), j=1,2$. If $x \in X$ and $\left(\varphi f_{1}\right)(x) \neq 0$, then $\varphi(x) \neq 0$ and $f_{1}(x) \neq 0$. Hence $i(x) \notin \bar{C}\left(f_{1}\right) \cap \bar{C}\left(f_{2}\right)$ and $i(x) \in \bar{C}\left(f_{1}\right)$. Thus $f_{2}(x)=0$. Therefore, $\varphi f_{1} \perp \varphi f_{2}$ and it follows that either $T\left(\varphi f_{1}\right)(y)=0$ or $T\left(\varphi f_{2}\right)(y)=0$. Without loss of generality, we may assume that the former holds. Then $T\left(f_{1}-\varphi f_{1}\right)(y)=\left(T f_{1}\right)(y) \neq 0$. Also, if $x \in X$ and $\left(f_{1}-\varphi f_{1}\right)(x) \neq 0$, then $\varphi(x) \neq 1$ and hence $i(x) \in{\overline{V_{2}}}^{\mathcal{A} X}$. Thus $\bar{C}\left(f_{1}-\varphi f_{1}\right) \subseteq$ ${\overline{V_{2}}}^{\mathcal{A} X} \subseteq U_{1} \cap U_{2}$.

It is easy to see that $\tilde{Y}$ is an open subset of $Y$. Note that $\mathcal{O}_{y} \neq \emptyset$ if $y \in \tilde{Y}$. By compactness of $\mathcal{A} X, \bigcap\left\{\bar{U}^{\mathcal{A} X}: U \in \mathcal{O}_{y}\right\} \neq \emptyset$ if $y \in \tilde{Y}$.

Lemma 6. If $y \in \tilde{Y}$, then $\bigcap\left\{\bar{U}^{\mathcal{A X}}: U \in \mathcal{O}_{y}\right\}$ contains exactly one point.

Proof. Suppose that there are distinct points $x_{1}, x_{2}$ in $\bigcap\left\{\bar{U}^{\mathcal{A} X}: U \in \mathcal{O}_{y}\right\}$. Let $U$ and $V$ be disjoint open neighborhoods of $x_{1}$ and $x_{2}$ respectively in $\mathcal{A} X$. Let $U_{1}$ and $V_{1}$ be open neighborhoods of $x_{1}$ and $x_{2}$ respectively in $\mathcal{A} X$ so that ${\overline{U_{1}}}^{\mathcal{A} X} \subseteq U$ and $\bar{V}_{1} \mathcal{A} X \subseteq V$. By Proposition 3, there exists $\varphi \in A(X)$ such that $\varphi\left(i^{-1}\left(U_{1}\right)\right) \subseteq\{0\}$ and $\varphi\left(i^{-1}\left(V_{1}\right)\right) \subseteq\{1\}$. For any $f \in A(X, E), \bar{C}(\varphi f) \subseteq \mathcal{A} X \backslash U_{1}$ and $\bar{C}(f-\varphi f) \subseteq$ $\mathcal{A} X \backslash V_{1}$. In particular, $x_{1} \notin \bar{C}(\varphi f)$ and $x_{2} \notin \bar{C}(f-\varphi f)$. Thus there exists an open neighborhood $W$ of $\bar{C}(\varphi f)$ so that $x_{1} \notin \bar{W}^{\mathcal{A} X}$. It follows that $W \notin \mathcal{O}_{y}$ and hence $T(\varphi f)(y)=0$. Similarly, $T(f-\varphi f)(y)=0$. Hence $T f(y)=T(\varphi f)(y)+T(f-$ $\varphi f)(y)=0$ for all $f \in A(X, E)$, contrary to the fact that $y \in \tilde{Y}$.

For each $y \in \tilde{Y}$, define $h(y)$ to be the unique point in $\bigcap\left\{\bar{U}^{\mathcal{A} X}: U \in \mathcal{O}_{y}\right\}$. If $f \in A(X, E)$ and $h(y) \notin \bar{C}(f)$, then there exists an open set $U$ in $\mathcal{A} X$ so that $\bar{C}(f) \subseteq U$ and $h(y) \notin \bar{U}^{\mathcal{A} X}$. Thus $U \notin \mathcal{O}_{y}$. Hence $T f(y)=0$.

Lemma 7. The map $h: \tilde{Y} \rightarrow \mathcal{A} X$ is continuous. 
Proof. Fix $y_{0} \in \tilde{Y}$. Let $U$ be an open neighborhood of $x_{0}=h\left(y_{0}\right)$ in $\mathcal{A} X$. Let $V$ be an open neighborhood of $x_{0}$ in $\mathcal{A} X$ such that $\bar{V}^{\mathcal{A} X} \subseteq U$. Take $P=i^{-1}(\mathcal{A} X \backslash U)$ and $Q=i^{-1}(V)$. Then $\overline{i(P)}^{\mathcal{A} X} \cap \overline{i(Q)}^{\mathcal{A} X}=\emptyset$. Hence there exists $\varphi \in A(X)$ so that $\varphi(P) \subseteq\{0\}$ and $\varphi(Q) \subseteq\{1\}$. Let $f \in A(X, E)$ be such that $T f\left(y_{0}\right) \neq 0$. If $x \in Q$, then $(f-\varphi f)(x)=0$. Hence $x_{0} \notin \bar{C}(f-\varphi f)$. By the above, $T(f-\varphi f)\left(y_{0}\right)=0$. So $T(\varphi f)\left(y_{0}\right) \neq 0$. Choose a neighborhood $W$ of $y_{0}$ in $Y$ such that $T(\varphi f)(y) \neq 0$ for all $y \in W$. If $y \in W \cap \tilde{Y}$ and $h(y) \notin \bar{U}^{\mathcal{A X}}$, then $h(y) \notin \bar{C}(\varphi f)$. Hence $T(\varphi f)(y)=0$, a contradiction. So we must have $h(W \cap \tilde{Y}) \subseteq \bar{U}^{\mathcal{A} X}$. This proves that $h$ is continuous on $\tilde{Y}$.

In the sequel, we will be concerned with operators that are continuous with respect to certain topologies. A useful consequence of it is that the map $h$ maps $\tilde{Y}$ into $i(X)$. A linear topology on $A(X, E)$ is said to be compactly determined if for every neighborhood $U$ of 0 in $A(X, E)$, there is a compact subset $K$ of $X$ such that $f \in U$ for all $f \in A(X, E)$ with $f_{\mid K}=0$.

Proposition 8. Suppose that $T: A(X, E) \rightarrow A(Y, F)$ is linear and separating. Assume that $T$ is continuous with respect to a compactly determined linear topology on $A(X, E)$ and a linear topology on $A(Y, F)$ that is stronger than the topology of pointwise convergence. Let $h: \tilde{Y} \rightarrow \mathcal{A} X$ be the support map. Then $h(\tilde{Y}) \subseteq i(X)$.

Proof. Suppose on the contrary that there exists $y_{0} \in \tilde{Y}$ such that $h\left(y_{0}\right)=x_{0} \notin$ $i(X)$. There exists $f_{0} \in A(X, E)$ such that $T f_{0}\left(y_{0}\right) \neq 0$. By assumption, there is a neighborhood $V$ of 0 in $A(Y, F)$ such that $\left\|g\left(y_{0}\right)\right\|<\left\|T f_{0}\left(y_{0}\right)\right\|$ for all $g \in V$. By continuity of $T$, there is a neighborhood $U$ of 0 in $A(X, E)$ so that $T U \subseteq V$. Since the topology on $A(X, E)$ is compactly determined, there exists a compact set $K$ in $X$ so that $f \in U$ for all $f \in A(X, E)$ with $f_{\mid K}=0$. Now, $x_{0}$ does not lie within the compact set $i(K)$. Hence there is an open neighborhood $\widetilde{Q}$ of $x_{0}$ in $\mathcal{A} X$ such that $\overline{\widetilde{Q}}^{\mathcal{A} X} \cap i(K)=\emptyset$. Let $Q=i^{-1}(\widetilde{Q}) \subseteq X$. Then

$$
\overline{i(K)}^{\mathcal{A} X} \cap \overline{i(Q)}^{\mathcal{A} X} \subseteq i(K) \cap \overline{\widetilde{Q}}^{\mathcal{A} X}=\emptyset .
$$

By Proposition 3, there exists $\varphi \in A(X)$ such that $\varphi(K) \subseteq\{0\}$ and $\varphi(Q) \subseteq\{1\}$. Consider the function $\varphi f_{0} \in A(X, E)$. We claim that $x_{0} \notin \bar{C}\left(f_{0}-\varphi f_{0}\right)$. For otherwise, there exists $x \in \widetilde{Q} \cap i\left(C\left(f_{0}-\varphi f_{0}\right)\right) \subseteq \widetilde{Q} \cap i(X)$. Then $\left(f_{0}-\varphi f_{0}\right)\left(i^{-1}(x)\right) \neq$ 0 and $x \in \widetilde{Q}$. This contradicts the fact that $i^{-1}(x) \in i^{-1}(\widetilde{Q})=Q$. Thus the claim is verified. By Proposition $4 T\left(\varphi f_{0}\right)\left(y_{0}\right)=T f_{0}\left(y_{0}\right)$. But since $\varphi f_{0 \mid K}=0$, $T\left(\varphi f_{0}\right) \in V$. Consequently, $\left\|T\left(\varphi f_{0}\right)\left(y_{0}\right)\right\|<\left\|T f_{0}\left(y_{0}\right)\right\|$ by choice of $V$. This contradiction completes the proof of the proposition.

\section{Continuous separating maps Between SPACES OF DIFFERENTIABLE FUNCTIONS}

From here on we will focus our attention on spaces of differentiable functions. Let $X$ and $Y$ be open subsets of Banach spaces $G$ and $H$ respectively. Assume that $1 \leq p \leq \infty, 0 \leq q \leq \infty$ and let $E$ and $F$ be Banach spaces. The space $C^{p}(X, E)$ consists of all functions $f: X \rightarrow E$ so that for all $k \in \mathbb{N}_{p}=\{i \in \mathbb{N} \cup\{0\}, i \leq p\}$, the (Fréchet) derivative $D^{k} f$ is a continuous function from $X$ into $\mathcal{S}^{k}(G, E)$, the space of all bounded symmetric $k$-linear maps from $G^{k}$ into $E$. (For notational convenience, we let $D^{0} f=f$ and $\mathcal{S}^{0}(G, E)=E$.) We will assume that there is a 
bump function $\varphi \in C^{p}(G)=C^{p}(G, \mathbb{R})$ so that $\varphi(x)=1$ if $\|x\| \leq 1 / 2, \varphi(x)=0$ if $\|x\| \geq 1$, and that $\sup _{x \in G}\left\|D^{k} \varphi(x)\right\|<\infty$ for all $k \in \mathbb{N}_{p}$. Such is the case, for example, if $G$ is a subspace of $\ell^{r}, p<r<\infty$, or a subspace of $\ell^{2 n}$ for some $n \in \mathbb{N}$. Refer to [6] for information on smoothness in Banach spaces. The space $C^{p}(X)$ satisfies property $(*)$ from $\S 1$ and, with the assumed existence of bump functions as above, it separates points from closed sets.

Suppose that $T: C^{p}(X, E) \rightarrow C^{q}(Y, F)$ is a linear separating map. If we take $A(X, E)=C^{p}(X, E)$ and $A(X)=C^{p}(X)$, then Proposition 4 applies to $T$. Furthermore, it is clear that the set $\tilde{Y}$ given by Proposition 4 is an open subset of $Y$ and thus an open subset of $H$. In place of $T$, it suffices to consider the map from $C^{p}(X, E)$ into $C^{q}(\tilde{Y}, F)$ given by $f \mapsto T f_{\mid \tilde{Y}}$. Without loss of generality, we may assume that $Y=\tilde{Y}$; equivalently, for all $y \in Y$, there exists $f \in C^{p}(X, E)$ so that $T f(y) \neq 0$. We call such an operator $T$ nowhere trivial. by

For each compact subset $K$ of $X$ and each $k \in \mathbb{N}_{p}, \rho_{K, k}: C^{p}(X, E) \rightarrow \mathbb{R}$ given

$$
\rho_{K, k}(f)=\sup _{x \in K} \sup _{0 \leq j \leq k}\left\|D^{j} f(x)\right\|
$$

defines a seminorm on $C^{p}(X, E)$. The set of seminorms $\rho_{K, k}$, where $K$ is a compact subset of $X$ and $k \in \mathbb{N}_{p}$, generates a compactly determined topology that is stronger than the topology of pointwise convergence. From now on, endow $C^{p}(X, E)$ with this topology and $C^{q}(Y, F)$ with the corresponding topology. In particular, assuming that $T: C^{p}(X, E) \rightarrow C^{q}(Y, F)$ is linear, separating and continuous, Proposition 8 applies. In this situation, we identify $i(X)$ with $X$ and consider the support map $h$ as a (continuous) map from $Y$ into $X$.

Proposition 9. Let $T: C^{p}(X, E) \rightarrow C^{q}(Y, F)$ be a separating, nowhere trivial and continuous linear operator and denote by $h$ the support map. For any $y_{0} \in Y$, there exist $r>0$ and $k_{0} \in \mathbb{N}_{p}$ such that $B\left(y_{0}, r\right) \subseteq Y$ and for all $y \in B\left(y_{0}, r\right), T f(y)=0$ for all $f \in C^{p}(X, E)$ with $D^{m} f(h(y))=0$ for all $0 \leq m \leq k_{0}$.

Proof. If the proposition fails, there is a sequence $\left(y_{k}\right)$ in $Y$ converging to $y_{0}$ and a sequence of functions $\left(f_{k}\right)$ in $C^{p}(X, E)$ so that $D^{m} f_{k}\left(h\left(y_{k}\right)\right)=0,0 \leq m \leq$ $\min \{k, p\}$, but $T f_{k}\left(y_{k}\right) \neq 0$. The set $K=\left\{y_{k}: k \in \mathbb{N} \cup\{0\}\right\}$ is compact. By continuity of $T$, there exist a compact subset $L$ of $X, k_{0} \in \mathbb{N}_{p}$, and $\delta>0$ such that $\rho_{L, k_{0}}(f) \leq \delta$ implies $\rho_{K, 0}(T f) \leq 1$. Consider the function $f=f_{k_{0}} \in C^{p}(X, E)$ and set $x_{0}=h\left(y_{k_{0}}\right)$. Let $\varphi \in C^{p}(G)$ be the bump function described above and let $C_{i}=\sup _{x \in G}\left\|D^{i} \varphi(x)\right\|<\infty$ for each $i$. Fix $\varepsilon>0$. Choose $\eta>0$ so that $\eta 2^{k_{0}} \max _{0 \leq i \leq k_{0}} C_{i} \leq \delta \varepsilon$. Since $D^{m} f\left(x_{0}\right)=0,0 \leq m \leq k_{0}$,

$$
\lim _{x \rightarrow x_{0}} \frac{\left\|D^{i} f(x)\right\|}{\left\|x-x_{0}\right\|^{k_{0}-i}}=0
$$

for $0 \leq i \leq k_{0}$. Choose $n \in \mathbb{N}$ so that $\left\|D^{i} f(x)\right\| \leq \eta\left\|x-x_{0}\right\|^{k_{0}-i}$ if $\left\|x-x_{0}\right\| \leq 1 / n$ and $0 \leq i \leq k_{0}$. Set $g(x)=\varphi\left(n\left(x-x_{0}\right)\right) f(x)$ for $x \in X$. Then $g \in C^{p}(X, E)$ and $x_{0} \notin \bar{C}(g-f)$. By Proposition 4. $T f\left(y_{k_{0}}\right)=T g\left(y_{k_{0}}\right)$. Note that $g(x)=0$ for all $x \in X$ with $\left\|x-x_{0}\right\| \geq 1 / n$. Thus $D^{m} g(x)=0$ for all $x \in X,\left\|x-x_{0}\right\|>1 / n$. On 
the other hand, for any $x \in X$ with $\left\|x-x_{0}\right\| \leq 1 / n$ and any $0 \leq m \leq k_{0}$,

$$
\begin{aligned}
\left\|D^{m} g(x)\right\| & \leq \sum_{i=0}^{m} n^{i}\left(\begin{array}{c}
m \\
i
\end{array}\right)\left\|D^{i} \varphi\left(n\left(x-x_{0}\right)\right)\right\|\left\|D^{m-i} f(x)\right\| \\
& \leq \sum_{i=0}^{m} n^{i}\left(\begin{array}{c}
m \\
i
\end{array}\right) C_{i}\left\|D^{m-i} f(x)\right\| \\
& \leq \sum_{i=0}^{m} n^{i}\left(\begin{array}{c}
m \\
i
\end{array}\right) C_{i} \eta\left\|x-x_{0}\right\|^{k_{0}-m+i} \\
& \leq \eta 2^{m} \max _{0 \leq i \leq m} C_{i} \leq \delta \varepsilon .
\end{aligned}
$$

By choice of $\delta$, it follows that $\rho_{K, 0}(T g) \leq \varepsilon$. In particular, $\left\|T f\left(y_{k_{0}}\right)\right\|=\left\|T g\left(y_{k_{0}}\right)\right\| \leq$ $\varepsilon$. Hence $T f\left(y_{k_{0}}\right)=0$, yielding a contradiction.

Remark. By considering the seminorm $\rho_{K, j}$ in place of $\rho_{K, 0}$, the proof shows that if $D^{m} f(h(y))=0$ for all $m \in \mathbb{N}_{p}$, then $D^{j}(T f)(y)=0$ for all $j \in \mathbb{N}_{q}$.

We now obtain a representation theorem for continuous linear separating maps between spaces of differentiable functions. For any $e \in G$, let $e^{k}$ be the element $(e, \ldots, e)$ ( $k$ components) in $G^{k}$.

Theorem 10. Let $T: C^{p}(X, E) \rightarrow C^{q}(Y, F)$ be a separating, nowhere trivial and continuous linear operator and denote by $h$ the support map. There exist functions $\Phi_{k}: Y \times \mathcal{S}^{k}(G, E) \rightarrow F, k \in \mathbb{N}_{p}$, so that for all $f \in C^{p}(X, E)$ and all $y \in Y$,

$$
T f(y)=\sum_{k} \Phi_{k}\left(y, D^{k} f(h(y))\right) .
$$

For each $y \in Y, \Phi_{k}(y, \cdot): \mathcal{S}^{k}(G, E) \rightarrow F$ is a bounded linear operator and, for each $S \in \mathcal{S}^{k}(G, E), \Phi_{k}(\cdot, S): Y \rightarrow F$ is continuous on $Y$. Furthermore, for every $y_{0} \in Y$, there exist $r>0$ and $k_{0} \in \mathbb{N}_{p}$ such that $\Phi_{k}(y, \cdot)=0$ for all $k>k_{0}$ and all $y \in B\left(y_{0}, r\right)$, and that $\left\{\Phi_{k}(y, \cdot): y \in B\left(y_{0}, r\right)\right\}$ is uniformly bounded for each $k \leq k_{0}$.

Proof. For any $y \in Y$ and any $S \in \mathcal{S}^{k}(G, E), k \in \mathbb{N}_{p}$, define the function $f_{y, S}$ : $X \rightarrow E$ by $f_{y, S}(x)=S\left[(x-h(y))^{k}\right]$. (If $k=0$, we take the right-hand side to mean simply $S$.) Let $\Phi_{k}: Y \times \mathcal{S}^{k}(G, E) \rightarrow F$ be given by $\Phi_{k}(y, S)=T f_{y, S}(y) / k$ !. For $y_{0} \in Y$, let $r_{1}>0$ and $k_{0} \in \mathbb{N}_{p}$ be given by Proposition 9 , Let $\bar{y} \in B\left(y_{0}, r_{1}\right)$ and $\bar{x}=h(\bar{y})$. If $k>k_{0}$, then, for any $S, D^{m} f_{\bar{y}, S}(\bar{x})=0,0 \leq m \leq k_{0}$. Hence $\Phi_{k}(\bar{y}, S)=0$ by Proposition 9 , For any $f \in C^{p}(X, E)$, consider the function

$$
P(x)=\sum_{k=0}^{k_{0}} \frac{D^{k} f(\bar{x})(x-\bar{x})^{k}}{k !} .
$$

Then $P \in C^{p}(X, E)$ and $D^{m}(f-P)(\bar{x})=0$ for $0 \leq m \leq k_{0}$. By choice of $k_{0}$, we have

$$
T f(\bar{y})=T P(\bar{y})=\sum_{k=0}^{k_{0}} \Phi_{k}\left(\bar{y}, D^{k} f(\bar{x})\right) .
$$

This gives rise to the representation (2) since $\Phi_{k}(\bar{y}, \cdot)=0$ for all $k>k_{0}$. The linearity of $\Phi_{k}(y, \cdot)$ is clear from the definition and the linearity of $T$. By direct 
computation, for any $y \in Y, S \in \mathcal{S}^{k}(G, E)$ and $x \in X$,

$$
D^{m} f_{y, S}(x)\left(a_{1}, \ldots, a_{m}\right)=\frac{k !}{(k-m) !} S\left((x-h(y))^{k-m}, a_{1}, \ldots, a_{m}\right)
$$

for all $a_{1}, \ldots, a_{m} \in G$ if $m \leq k$, and $D^{m} f_{y, S}=0$ if $m>k$. Let $L$ be a compact subset of $Y$. By continuity of $T$, there exist a compact $K \subseteq X, k \in \mathbb{N}_{p}$ and $C<\infty$ so that $\rho_{L, 0}(T f) \leq C \rho_{K, k}(f)$ for all $f \in C^{p}(X, E)$. From the expression for $D^{m} f_{y, S}$, we see that

$$
\sup \left\{\rho_{K, k}\left(f_{y, S}\right): y \in L, S \in \mathcal{S}^{k}(G, E),\|S\| \leq 1\right\}<\infty .
$$

It follows that

$$
\sup \left\{\left\|T f_{y, S}(y)\right\|: y \in L,\|S\| \leq 1\right\} \leq \sup \left\{\rho_{L, 0}\left(T f_{y, S}\right): y \in L,\|S\| \leq 1\right\}<\infty .
$$

This shows that each $\Phi_{k}(y, \cdot)$ is a bounded linear operator and that $\left\{\Phi_{k}(y, \cdot): y \in\right.$ $L\}$ is uniformly bounded on any compact subset $L$ of $Y$. If there exists $y_{0} \in Y$ such that $\left\{\Phi_{k}(y, \cdot): y \in B\left(y_{0}, r\right)\right\}$ is not uniformly bounded for any $r>0$, then there is a sequence $\left(y_{n}\right)$ converging to $y_{0}$ so that $\left(\Phi_{k}\left(y_{n}, \cdot\right)\right)$ is unbounded. This contradicts the above since the set $\left\{\left(y_{n}\right)\right\} \cup\left\{y_{0}\right\}$ is compact in $Y$. Similarly, if $S$ is bounded, it follows easily that if $\left(y_{n}\right)$ converges to $y$ in $Y$, then $\left(D^{m} f_{y_{n}, S}\right)$ converges uniformly to $D^{m} f_{y, S}$ on any compact subset $K$ of $X$ for any $m$. Thus $\left(f_{y_{n}, S}\right)$ converges to $f_{y, S}$ in $C^{p}(X, E)$. Hence $\left(T f_{y_{n}, S}\right)$ converges uniformly to $T f_{y, S}$ on the compact set $\left\{\left(y_{n}\right)\right\} \cup\{y\}$. Therefore, $\left(\Phi_{k}\left(y_{n}, S\right)\right)$ converges to $\Phi_{k}(y, S)$. This establishes the continuity of $\Phi_{k}(\cdot, S)$.

Theorem 10 is not the last word since there is no mention of differentiability of $h$ or $\Phi_{k}$. In the next few sections, we will see that in some cases (depending on $p$ and $q$ ), weak compactness of $T$ leads to degeneracy (constancy on connected components) of the support map.

\section{Degeneracy of the SUpport map, $q>p$}

This section and the next two are at the heart of the paper. From here on, $T$ : $C^{p}(X, E) \rightarrow C^{q}(Y, F)$ will always be a separating, nowhere trivial and continuous linear operator, having a representation given by Theorem 10. The support map $h$ is implicitly determined by the operator $T$ via the representation given in Theorem 10. We want to extract information on $h$ under various assumptions on $T$. We begin with the simplest case in this section; namely, when $q>p$. Recall that we assume the existence of a bump function $\varphi$ with bounded derivatives on the Banach space $G$ containing $X$. Let $C_{j}=\sup _{x \in G}\left\|D^{j} \varphi(x)\right\|$.

Lemma 11. Let $\left(x_{n}\right)$ be a sequence in $X$ converging to a point $x_{0} \in X$. Suppose that $\left\|x_{n}-x_{0}\right\|=r_{n}$, and that $0<3 r_{n+1}<r_{n}$ for all $n \in \mathbb{N}$. Set $\varphi_{n}(x)=$ $\varphi\left(\frac{2}{r_{n}}\left(x-x_{n}\right)\right)$. Let $\left(f_{n}\right)$ be a sequence in $C^{p}(X, E)$ and let

$$
\eta_{n k}=\sum_{j=0}^{k}\left(\begin{array}{c}
k \\
j
\end{array}\right) \frac{C_{j}}{r_{n}^{j}} \sup _{x \in B\left(x_{n}, r_{n} / 2\right)}\left\|D^{k-j} f_{n}(x)\right\|
$$

for all $n \in \mathbb{N}$ and all $k \in \mathbb{N}_{p}$. If

$$
\lim _{n \rightarrow \infty} \frac{\eta_{n k}}{r_{n}}=0, \quad 0 \leq k<p
$$


and

$$
\lim _{n \rightarrow \infty} \eta_{n p}=0, \quad \text { in case } p<\infty,
$$

then the pointwise sum $f=\sum \varphi_{n} f_{n}$ belongs to $C^{p}(X, E)$, and $D^{k} f\left(x_{0}\right)=0, k \in \mathbb{N}_{p}$.

Proof. Since the supports of the functions $\varphi_{n} f_{n}$ tend toward the point $x_{0}$, it is clear that $f$ is $C^{p}$ on $X \backslash\left\{x_{0}\right\}$. We first prove by induction that $D^{k} f\left(x_{0}\right)=0$ for all $k \in \mathbb{N}_{p}$. The case $k=0$ is trivial. Assume that the claim has been verified for some $k, 0 \leq k<p$. If $x \notin \bigcup B\left(x_{n}, r_{n} / 2\right)$, then $D^{k} f(x)=0$. Suppose that $x \in B\left(x_{n}, r_{n} / 2\right)$ for some $n$. Then

$$
\begin{aligned}
\left\|D^{k} f(x)-D^{k} f\left(x_{0}\right)\right\| & =\left\|D^{k} f(x)\right\|=\left\|D^{k}\left(\varphi_{n} f_{n}\right)(x)\right\| \\
& \leq \sum_{j=0}^{k}\left(\begin{array}{c}
k \\
j
\end{array}\right)\left\|D^{j} \varphi_{n}(x)\right\|\left\|D^{k-j} f_{n}(x)\right\| \\
& \leq \sum_{j=0}^{k}\left(\begin{array}{c}
k \\
j
\end{array}\right) \frac{2^{j} C_{j}}{r_{n}^{j}}\left\|D^{k-j} f_{n}(x)\right\| \leq 2^{k} \eta_{n k} .
\end{aligned}
$$

Also, $\left\|x-x_{0}\right\| \geq \frac{r_{n}}{2}$ for all $x \in B\left(x_{n}, r_{n} / 2\right)$. It follows from condition (3) that $D^{k+1} f\left(x_{0}\right)=0$. If $p<\infty$, we need to show that $D^{p} f$ is continuous at $x_{0}$. But the same computation shows that $\left\|D^{p} f(x)-D^{p} f\left(x_{0}\right)\right\| \leq 2^{p} \eta_{n p}$ if $x \in B\left(x_{n}, r_{n} / 2\right)$ for some $n$ and 0 otherwise. The continuity of $D^{p} f$ at $x_{0}$ follows from condition (4).

Theorem 12. Assume that $1 \leq p<\infty$ and that at $y_{0} \in Y, \Phi_{i}\left(y_{0}, \cdot\right) \neq 0$ for some $i \leq p$. For all $q^{\prime} \in \mathbb{N}_{q}$, there exist $r>0$ and $C<\infty$ so that $\left\|h(y)-h\left(y_{0}\right)\right\|^{p-i} \leq$ $C\left\|y-y_{0}\right\|^{q^{\prime}}$ for all $y \in B\left(y_{0}, r\right)$.

Proof. Otherwise, there are a sequence $\left(y_{n}\right)$ in $Y \backslash\left\{y_{0}\right\}$ converging to $y_{0}$ and $q^{\prime} \in \mathbb{N}_{q}$ so that $\left(\left\|h\left(y_{n}\right)-h\left(y_{0}\right)\right\|^{p-i} /\left\|y_{n}-y_{0}\right\|^{q^{\prime}}\right)$ diverges to $\infty$. Hence it is possible to choose a positive sequence $\left(c_{n}\right)$ so that $c_{n} /\left\|h\left(y_{n}\right)-h\left(y_{0}\right)\right\|^{p-i} \rightarrow 0$ and $c_{n} /\left\|y_{n}-y_{0}\right\|^{q^{\prime}} \rightarrow \infty$. Set $x_{n}=h\left(y_{n}\right)$ and $x_{0}=h\left(y_{0}\right)$. By using a subsequence if necessary, we may further assume that $0<3 r_{n+1}<r_{n}$, where $r_{n}=\left\|x_{n}-x_{0}\right\|$. Let $S \in \mathcal{S}^{i}(G, E)$ be such that $\Phi_{i}\left(y_{0}, S\right) \neq 0$. Define $f_{n}(x)=c_{n} S\left(x-x_{n}\right)^{i}$ and $\varphi_{n}$ as in Lemma 11. By choice of $c_{n}, f=\sum \varphi_{n} f_{n}$ lies in $C^{p}(X, E)$ and $D^{j} f\left(x_{0}\right)=0$ for all $j \in \mathbb{N}_{p}$. Thus $T f$ is $C^{q}$ and, a fortiori, lies in $C^{q^{\prime}}$. Furthermore, by the Remark following Proposition 9, $D^{j}(T f)\left(y_{0}\right)=0$ for $0 \leq j \leq q^{\prime}$. Hence $\lim _{y \rightarrow y_{0}} T f(y) /\left\|y-y_{0}\right\|^{q^{\prime}}=0$. Since $T f\left(y_{n}\right)=c_{n} i ! \Phi_{i}\left(y_{n}, S\right)$ and $\left(\Phi_{i}\left(y_{n}, S\right)\right)$ converges to $\Phi_{i}\left(y_{0}, S\right) \neq 0$, we have $c_{n} /\left\|y_{n}-y_{0}\right\|^{q^{\prime}} \rightarrow 0$, contrary to the choice of $c_{n}$.

Corollary 13. Let $T: C^{p}(X, E) \rightarrow C^{q}(Y, F)$ be a separating, nowhere trivial continuous linear operator. If $q>p$, then there is a partition of $Y$ into clopen subsets $\left(Y_{\alpha}\right)$ so that the support map is constant on each $Y_{\alpha}$.

Proof. Choose $q^{\prime} \in \mathbb{N}_{q}$ so that $q^{\prime}>p$. Since $T$ is nowhere trivial, for any $y_{0} \in Y$, there exists $i \in \mathbb{N}_{p}$ so that $\Phi_{i}\left(y_{0}, \cdot\right) \neq 0$. Observe that $q^{\prime}>p-i$. By Theorem 12 ,

$$
\limsup _{y \rightarrow y_{0}} \frac{\left\|h(y)-h\left(y_{0}\right)\right\|}{\left\|y-y_{0}\right\|} \leq C \lim _{y \rightarrow y_{0}}\left\|y-y_{0}\right\|^{\frac{q^{\prime}}{p-i}-1}=0 .
$$

Thus $D h(y)=0$ for all $y \in Y$ and the conclusion of the corollary follows. 


\section{Degeneracy of the support map, $p=q=\infty$}

Certainly, for different arrangements of $p$ and $q$, and without additional assumptions, degeneracy of the support map will not result. Recall that a set $W$ in $C^{q}(Y, F)$ is said to be bounded if it is absorbed by every open neighborhood of 0 ; equivalently, if each seminorm $\rho_{L, \ell}$ (see (1)) is bounded on $W$. In this section, we show that when $p=q=\infty$ and $T$ maps some open set onto a bounded set, then the support map is degenerate. The condition on $T$ is satisfied in particular when it is compact or weakly compact.

Lemma 14. For any $\ell \in \mathbb{N}$, there exists $c<\infty$ so that given $a \in X, 0<\varepsilon<1$ with $B(a, \varepsilon) \subseteq X$ and $f \in C^{\infty}(X, E)$ with $D^{j} f(a)=0,0 \leq j \leq \ell$, there exists $g \in C^{\infty}(X, E)$ such that $g=f$ on $B(a, \varepsilon)$ and

$$
\max _{0 \leq j \leq \ell} \sup _{x \in X}\left\|D^{j} g(x)\right\| \leq c \sup _{x \in B(a, 2 \varepsilon)}\left\|D^{\ell} f(x)\right\| .
$$

Proof. By translation, we may assume that $a=0$. As usual, let $\varphi$ denote the assumed bump function and set $c=2^{\ell} \max _{0 \leq k \leq \ell} \sup _{x \in G}\left\|D^{k} \varphi(x)\right\|$. Given $\varepsilon>0$ with $B(0, \varepsilon) \subseteq X$, consider the function $g: X \rightarrow E$ defined by $g(x)=\varphi\left((2 \varepsilon)^{-1} x\right) f(x)$. Obviously, $g \in C^{\infty}(X, E)$ and $g=f$ on $B(0, \varepsilon)$. Since $g(x)=0$ when $\|x\|>2 \varepsilon$, $D^{j} g(x)=0$ for $\|x\|>2 \varepsilon$ and any $j \in \mathbb{N} \cup\{0\}$. If $x \in X$ and $\|x\| \leq 2 \varepsilon$, by Taylor's Theorem (see, e.g., [14]) and using the fact that $D^{j} f(0)=0,0 \leq j \leq \ell$, we have, for $0 \leq k \leq \ell$,

$$
\begin{aligned}
\left\|D^{k} f(x)\right\| & \leq \int_{0}^{1} \frac{(1-t)^{\ell-k-1}}{(\ell-k-1) !}\left\|D^{\ell} f(t x)\right\|\|x\|^{\ell-k} d t \\
& \leq \sup _{z \in B(0,2 \varepsilon)}\left\|D^{\ell} f(z)\right\| \frac{(2 \varepsilon)^{\ell-k}}{(\ell-k) !} .
\end{aligned}
$$

Thus, for $0 \leq j \leq \ell$,

$$
\begin{aligned}
\left\|D^{j} g(x)\right\| & \leq \sum_{k=0}^{j}\left(\begin{array}{l}
j \\
k
\end{array}\right)\left(\frac{1}{2 \varepsilon}\right)^{j-k}\left\|D^{j-k} \varphi\left(\frac{x}{2 \varepsilon}\right)\right\|\left\|D^{k} f(x)\right\| \\
& \leq \sum_{k=0}^{j}\left(\begin{array}{l}
j \\
k
\end{array}\right)(2 \varepsilon)^{\ell-j}\left\|D^{j-k} \varphi\left(\frac{x}{2 \varepsilon}\right)\right\| \sup _{z \in B(0,2 \varepsilon)}\left\|D^{\ell} f(z)\right\| \\
& \leq c \sup _{z \in B(0,2 \varepsilon)}\left\|D^{\ell} f(z)\right\| .
\end{aligned}
$$

For the remainder of the section, fix a separating, nowhere trivial linear operator $T: C^{\infty}(X, E) \rightarrow C^{\infty}(Y, F)$ which maps some open set in $C^{\infty}(X, E)$ onto a bounded set in $C^{\infty}(Y, F)$. In particular, $T$ is continuous. Given $y_{0} \in Y$, we may find by applying Theorem 10, $k_{0} \in \mathbb{N}$ and $r>0$ so that

$$
T f(y)=\sum_{k=0}^{k_{0}} \Phi_{k}\left(y, D^{k} f(h(y))\right), y \in B\left(y_{0}, r\right) \subseteq Y, f \in C^{\infty}(X, E) .
$$

Moreover, since $T$ maps an open set onto a bounded set, there are a compact set $L \subseteq X$ and $\ell \in \mathbb{N}$ such that for all $m \in \mathbb{N} \cup\{0\}$ and every compact $K \subseteq Y$, there 
exists $C=C(K, m)<\infty$ so that

$$
\max _{y \in K}\left\|D^{m}(T f)(y)\right\| \leq C \max _{0 \leq j \leq \ell} \max _{x \in L}\left\|D^{j} f(x)\right\| .
$$

We may assume without loss of generality that $\ell \geq k_{0}$.

Proposition 15. If $f \in C^{\infty}(X, E)$ and $D^{j} f(h(y))=0,0 \leq j \leq \ell$, for some $y \in B\left(y_{0}, r\right)$, then $D^{m}(T f)(y)=0, m \in \mathbb{N} \cup\{0\}$.

Proof. By Lemma 14, for any $\delta>0$, there exists $g \in C^{\infty}(X, E)$ so that $g=f$ on a neighborhood of $h(y)$ and $\max _{0 \leq j \leq \ell} \sup _{x \in X}\left\|D^{j} g(x)\right\| \leq \delta$. By the representation of $T, T g=T f$ on a neighborhood of $y$. From (5), we deduce that $\left\|D^{m}(T f)(y)\right\|=$ $\left\|D^{m}(T g)(y)\right\| \leq C(\{y\}, m) \delta$. The result follows since $\delta>0$ is arbitrary.

Denote $h\left(y_{0}\right)$ by $a$ and assume that $B(a, s) \subseteq X$ for some $0<s<1$.

Proposition 16. Let $K$ be a compact convex set in $B\left(y_{0}, r\right)$ containing the point $y_{0}$. Suppose that $0 \leq j \leq k_{0}, S \in \mathcal{S}^{j}(G, E)$ and $r_{1}, r_{2}, r_{3}, r_{4}$ are nonnegative integers so that $r_{1}+r_{3}=\ell+1, r_{3}+r_{4}=j$ and $\alpha=r_{2}+r_{4} \leq k_{0}$. If $z \in K$, $b=h(z) \in B(a, s)$, and $x^{*}$ is a norm 1 functional in the dual space $G^{*}$ of $G$ such that $x^{*}(b-a)=\|b-a\|$, define $f: X \rightarrow E$ by

$$
f(x)=\left(x^{*}(x-a)\right)^{r_{1}}\left(x^{*}(x-b)\right)^{r_{2}} S(x-a)^{r_{3}}(x-b)^{r_{4}} .
$$

Then there exists a finite constant $D=D(K)$ such that

$$
\|T f(z)\| \leq D\|S\|\|b-a\|^{\alpha+1}\left\|z-y_{0}\right\|^{\ell+1} .
$$

Proof. Since $r_{1}+r_{3}>\ell, D^{j} f(a)=0,0 \leq j \leq \ell$. Consider any $\varepsilon$ so that $\|b-a\|<$ $\varepsilon<s$. By Lemma 14, there exists $g \in C^{\infty}(X, E)$ such that $g=f$ on $B(a, \varepsilon)$ and

$$
\max _{0 \leq j \leq \ell} \sup _{x \in X}\left\|D^{j} g(x)\right\| \leq c \sup _{x \in B(a, 2 \varepsilon)}\left\|D^{\ell} f(x)\right\| .
$$

Now, for $x \in B(a, 2 \varepsilon)$,

$$
\begin{aligned}
\left\|D^{\ell} f(x)\right\| \leq \ell ! \sum \prod_{i=1}^{4}\left(\begin{array}{c}
r_{i} \\
s_{i}
\end{array}\right)\|x-a\|^{r_{1}-s_{1}}\|x-b\|^{r_{2}-s_{2}} \\
\cdot\|S\|\|\| x-a\left\|^{r_{3}-s_{3}}\right\| x-b \|^{r_{4}-s_{4}},
\end{aligned}
$$

where the sum is taken over all integers $0 \leq s_{i} \leq r_{i}, 1 \leq i \leq 4$, with sum $\ell$. Using the estimates $\|x-a\|,\|x-b\|<3 \varepsilon$ and

$$
\sum \prod_{i=1}^{4}\left(\begin{array}{c}
r_{i} \\
s_{i}
\end{array}\right) \leq \prod_{i=1}^{4} \sum_{s_{i}=0}^{r_{i}}\left(\begin{array}{c}
r_{i} \\
s_{i}
\end{array}\right) \leq 2^{\ell+1+\alpha} \leq 2^{2 \ell+1},
$$

we see that

$$
\left\|D^{\ell} f(x)\right\| \leq \ell ! 2^{2 \ell+1}\|S\|(3 \varepsilon)^{\alpha+1} .
$$

Take $C=C(K, \ell+1)$. From (5) and the above, we obtain

$$
\begin{aligned}
\max _{y \in K}\left\|D^{\ell+1}(T g)(y)\right\| & \leq C \max _{0 \leq j \leq \ell} \max _{x \in L}\left\|D^{j} g(x)\right\| \\
& \leq C c \sup _{x \in B(a, 2 \varepsilon)}\left\|D^{\ell} f(x)\right\| \\
& \leq C c \ell ! 2^{2 \ell+1} 3^{\ell+1}\|S\| \varepsilon^{\alpha+1} .
\end{aligned}
$$


By Proposition 15, $D^{m}(T g)\left(y_{0}\right)=0, m \in \mathbb{N} \cup\{0\}$. Since $K$ is convex, by Taylor's Theorem,

$$
\begin{aligned}
\|T g(z)\| & \leq \max _{y \in K}\left\|D^{\ell+1}(T g)(y)\right\| \frac{\left\|z-y_{0}\right\|^{\ell+1}}{(\ell+1) !} \\
& \leq C c 2^{2 \ell+1} 3^{\ell+1}\|S\| \varepsilon^{\alpha+1}\left\|z-y_{0}\right\|^{\ell+1} .
\end{aligned}
$$

Observe that $T f(z)=T g(z)$ as $f=g$ on a neighborhood of $h(z)=b$. Since we may make $\varepsilon$ as close to $\|b-a\|$ as we please, the result follows.

Proposition 17. In the notation of Proposition 16, let $W$ be the operator in $\mathcal{S}^{\alpha}(G, E)$ determined by

$$
W w^{\alpha}=\left(x^{*}(b-a)\right)^{r_{1}}\left(x^{*}(w)\right)^{r_{2}} S(b-a)^{r_{3}} w^{r_{4}} .
$$

Then

$$
\alpha !\left\|\Phi_{\alpha}(z, W)\right\| \leq 2^{\left(k_{0}-\alpha\right)(\ell+2)} D\|S\|\|b-a\|^{\alpha+1}\left\|z-y_{0}\right\|^{\ell+1} .
$$

Proof. We proceed by induction on $\alpha$, beginning with $\alpha=k_{0}$ and ending at $\alpha=0$. Suppose that $\alpha=k_{0}$. Let $f$ be the function defined in Proposition 16. Then

$$
\left\|\sum_{k=0}^{k_{0}} \Phi_{k}\left(z, D^{k} f(b)\right)\right\|=\|T f(z)\| \leq D\|S\|\|b-a\|^{\alpha+1}\left\|z-y_{0}\right\|^{\ell+1} .
$$

Since $r_{2}+r_{4}=\alpha=k_{0}, D^{k} f(b)=0$ if $k<k_{0}$, and $D^{k_{0}} f(b)=k_{0} ! W$. Hence

$$
k_{0} !\left\|\Phi_{k_{0}}(z, W)\right\| \leq D\|S\|\|b-a\|^{\alpha+1}\left\|z-y_{0}\right\|^{\ell+1} .
$$

Now suppose that $0 \leq \alpha<k_{0}$, and the proposition has been proved for any $\beta$, $\alpha<\beta \leq k_{0}$. Let $f$ be the function defined in Proposition 16, with the parameter $\alpha$. Then $D^{k} f(b)=0$ if $k<\alpha$. Hence

$$
\left\|\sum_{k=\alpha}^{k_{0}} \Phi_{k}\left(z, D^{k} f(b)\right)\right\|=\|T f(z)\| \leq D\|S\|\|b-a\|^{\alpha+1}\left\|z-y_{0}\right\|^{\ell+1} .
$$

For $\alpha \leq k \leq k_{0}$

$$
D^{k} f(b)=k ! \sum\left(\begin{array}{l}
r_{1} \\
s_{1}
\end{array}\right)\left(\begin{array}{l}
r_{3} \\
s_{3}
\end{array}\right) W_{s_{1}, s_{3}},
$$

where the sum is taken over all integers $0 \leq s_{1} \leq r_{1}, 0 \leq s_{3} \leq r_{3}$, such that $s_{1}+s_{3}=k-\alpha$, and, for each such pair,

$$
W_{s_{1}, s_{3}} w^{k}=\left(x^{*}(b-a)\right)^{r_{1}-s_{1}}\left(x^{*}(w)\right)^{s_{1}+r_{2}} S(b-a)^{r_{3}-s_{3}} w^{s_{3}+r_{4}} .
$$

If $k_{0} \geq k>\alpha$, the inductive hypothesis gives

$$
k !\left\|\Phi_{k}\left(z,\left(x^{*}(b-a)\right)^{k-\alpha} W_{s_{1}, s_{3}}\right)\right\| \leq 2^{\left(k_{0}-k\right)(\ell+2)} D\|S\|\|b-a\|^{k+1}\left\|z-y_{0}\right\|^{\ell+1} .
$$

Since $x^{*}(b-a)=\|b-a\|$ and $\sum\left(\begin{array}{l}r_{1} \\ s_{1}\end{array}\right)\left(\begin{array}{l}r_{3} \\ s_{3}\end{array}\right) \leq 2^{r_{1}+r_{3}}=2^{\ell+1}$, we get that

$$
\left\|\Phi_{k}\left(z, D^{k} f(b)\right)\right\| \leq 2^{\ell+1+\left(k_{0}-k\right)(\ell+2)} D\|S\|\|b-a\|^{\alpha+1}\left\|z-y_{0}\right\|^{\ell+1} .
$$


Then

$$
\begin{aligned}
\alpha !\left\|\Phi_{\alpha}(z, W)\right\| & =\left\|\Phi_{\alpha}\left(z, D^{\alpha} f(b)\right)\right\| \\
& \leq \sum_{k=\alpha+1}^{k_{0}}\left\|\Phi_{k}\left(z, D^{k} f(b)\right)\right\|+D\|S\|\|b-a\|^{\alpha+1}\left\|z-y_{0}\right\|^{\ell+1} \\
& \leq\left[\sum_{k=\alpha+1}^{k_{0}} 2^{\ell+1+\left(k_{0}-k\right)(\ell+2)}+1\right] \cdot D\|S\|\|b-a\|^{\alpha+1}\left\|z-y_{0}\right\|^{\ell+1} \\
& \leq 2^{\left(k_{0}-\alpha\right)(\ell+2)} D\|S\|\|b-a\|^{\alpha+1}\left\|z-y_{0}\right\|^{\ell+1},
\end{aligned}
$$

completing the induction.

Theorem 18. Let $T: C^{\infty}(X, E) \rightarrow C^{\infty}(Y, F)$ be a separating, nowhere trivial linear operator which maps some open set in $C^{\infty}(X, E)$ onto a bounded set in $C^{\infty}(Y, F)$. Then there is a partition of $Y$ into clopen subsets $\left(Y_{\alpha}\right)$ so that the support map is constant on each $Y_{\alpha}$.

Proof. Let $y_{0} \in Y$ and keep the foregoing notation. Since $T$ is nowhere trivial, there exist $0 \leq j \leq k_{0}$ and $S \in \mathcal{S}^{j}(G, E)$ so that $\Phi_{j}\left(y_{0}, S\right) \neq 0$. Choose $1>r, s>0$ so that $B\left(y_{0}, r\right) \subseteq Y, h\left(B\left(y_{0}, r\right)\right) \subseteq B\left(h\left(y_{0}\right), s\right) \subseteq X$ and $\inf _{z \in B\left(y_{0}, r\right)}\left\|\Phi_{j}(z, S)\right\|=$ $\delta>0$. Take any $z_{0} \in B\left(y_{0}, r\right) \backslash\left\{y_{0}\right\}$ and let $K$ be the compact interval $\left[y_{0}, z_{0}\right]$. Let $z \in K \backslash\left\{y_{0}\right\}, b=h(z)$ and let $x^{*}$ be a norming functional for $b-a=b-h\left(y_{0}\right)$. Apply Proposition [17 with $r_{1}=\ell+1, r_{2}=r_{3}=0$ and $r_{4}=j$. Note that $\alpha=j$ as well. We obtain, with $D=D(K)$,

$$
j !\left|x^{*}(b-a)^{\ell+1}\right|\left\|\Phi_{j}(z, S)\right\| \leq 2^{\left(k_{0}-j\right)(\ell+2)} D\|S\|\|b-a\|^{j+1}\left\|z-y_{0}\right\|^{\ell+1} .
$$

Hence

$$
\delta j !\left(\frac{\|b-a\|}{\left\|z-y_{0}\right\|}\right)^{\ell-j} \leq 2^{\left(k_{0}-j\right)(\ell+2)} D\|S\|\left\|z-y_{0}\right\|^{j+1} .
$$

Note that $j \leq k_{0} \leq \ell$. Since the right-hand side tends to 0 as $z$ tends to $y_{0}$ in $K$, $j \neq \ell$. Thus $\ell-j>0$. Therefore,

$$
\lim _{\substack{z \rightarrow y_{0} \\ z \in\left[y_{0}, z_{0}\right]}} \frac{h(z)-h\left(y_{0}\right)}{z-y_{0}}=0 .
$$

That is, $h$ has directional derivative 0 in every direction at any point $y_{0} \in Y$. It follows easily that $h$ must be constant on a neighborhood of every point in $Y$, from which the conclusion of the theorem is easily deduced.

Example. Suppose that $1 \leq q \leq p \leq \infty$ and that $q$ is finite. Then there may exist a separating, nowhere trivial linear operator $T: C^{p}(X, E) \rightarrow C^{q}(Y, F)$ which maps some open set in $C^{p}(X, E)$ onto a bounded set in $C^{q}(Y, F)$ whose support map is not locally constant. Indeed, let $I=(0,1)$ and consider the map $T: C^{p}(\mathbb{R}) \rightarrow C^{q}(I)$ defined by $T f=f_{\mid I}$. Clearly, $T$ is a separating, nowhere trivial linear operator. Taking $L=[0,1]$, it maps the open set $\left\{f \in C^{p}(\mathbb{R}): \rho_{L, q}(f)<1\right\}$ onto a bounded set in $C^{q}(I)$. However, it is clear that the support map is $h(y)=y$ for all $y \in I$.

Furthermore, if $q<p$, then $T$ maps the open set $\left\{f \in C^{p}(\mathbb{R}): \rho_{L, q+1}(f)<1\right\}$ onto a relatively compact set in $C^{q}(I)$. 


\section{Degeneracy of the support map, $p=q<\infty$}

The example in the last section shows that the methods of the previous two sections cannot be used to show that the support map of $T$ is locally constant when $p=q<\infty$, even if $T$ maps an open set onto a bounded set. However, we will show in this section that the support map is locally constant if the nowhere trivial, separating map $T: C^{p}(X, E) \rightarrow C^{p}(Y, F)$ is assumed to be weakly compact.

In this section, we assume that $1 \leq p<\infty$ and that $T: C^{p}(X, E) \rightarrow C^{p}(Y, F)$ is a weakly compact, separating, nowhere trivial linear map. Denote by $h$ the support map of $T$. By Theorem 10, $T$ has the representation

$$
T f(y)=\sum_{k=0}^{p} \Phi_{k}\left(y, D^{k} f(h(y))\right), y \in Y, f \in C^{p}(X, E) .
$$

Lemma 19. Suppose that there are $k_{0}, 0<k_{0} \leq p$ and $y_{0} \in Y$ such that $\Phi_{k_{0}}\left(y_{0}, \cdot\right) \neq 0$. Then $h$ is constant on a neighborhood of $y_{0}$.

Proof. By Theorem 12, there exist $r>0$ and $C<\infty$ so that $\left\|h(y)-h\left(y_{0}\right)\right\|^{p-k_{0}} \leq$ $\left\|y-y_{0}\right\|^{p}$ for all $y \in B\left(y_{0}, r\right)$. Hence $\lim _{y \rightarrow y_{0}}\left\|h(y)-h\left(y_{0}\right)\right\| /\left\|y-y_{0}\right\|=0$; that is, $D h\left(y_{0}\right)=0$. Fix $S$ such that $\Phi_{k_{0}}\left(y_{0}, S\right) \neq 0$. By continuity of $\Phi_{k_{0}}(\cdot, S)$, $\Phi_{k_{0}}(y, \cdot) \neq 0$ for all $y$ in a neighborhood of $y_{0}$. Hence $D h(y)=0$ for all $y$ in a neighborhood of $y_{0}$. The lemma follows.

A subset $Y^{\prime}$ of $Y$ is perfect if every point of $Y^{\prime}$ is an accumulation point of $Y^{\prime}$. If $f$ is a function from $Y^{\prime}$ into a Banach space, then we may define the derivative of $f$ in the usual manner, with the relevant limit taken in $Y^{\prime}$. Precisely, $f$ is differentiable at $y_{0} \in Y^{\prime}$ if there exists a bounded linear operator $A$ so that

$$
\lim _{\substack{y \rightarrow y_{0} \\ y \in Y^{\prime}}} \frac{\left\|f(y)-f\left(y_{0}\right)-A\left(y-y_{0}\right)\right\|}{\left\|y-y_{0}\right\|}=0 .
$$

The derivative operator $A$, if it exists, is denoted by $D_{Y^{\prime}} f\left(y_{0}\right)$. Higher derivatives may be defined in the same manner. The usual rules of differentiation, in particular the chain rule and product and quotient rules for real-valued functions, still hold.

Proposition 20. Suppose that $Y^{\prime}$ is a perfect subset of $Y$ and that $\Phi_{k}(y, \cdot)=0$ for all $y \in Y^{\prime}$ and all $k, 0<k \leq p$. Then for all $x^{*} \in G^{*}$ and all $y_{0} \in Y$, $D_{Y^{\prime}}\left(x^{*} \circ h\right)\left(y_{0}\right)=0$.

Proof. By the assumptions, $T$ has the representation $T f(y)=\Phi_{0}(y, f(h(y)))$ for all $y \in Y^{\prime}$. For any $y_{0} \in Y^{\prime}$, since $T$ is nowhere trivial, there exists $u \in E$ such that $\Phi_{0}\left(y_{0}, u\right) \neq 0$. Set $g(y)=\Phi_{0}(y, u), y \in Y^{\prime}$. Then $g$ is the restriction to $Y^{\prime}$ of a function in $C^{p}(Y, F)$. For any $\alpha \in C^{p}(X),(\alpha \circ h) \cdot g=T(\alpha \cdot u)_{\mid Y^{\prime}}$ is $p$ times continuously differentiable on $Y^{\prime}$. Choose $v^{*} \in F^{*}$ so that $v^{*} \circ g \neq 0$ on a neighborhood $O$ of $y_{0}$. Then $\alpha \circ h=v^{*} \circ T(\alpha \cdot u) / v^{*} \circ g$ on $O \cap Y^{\prime}$ and hence $\alpha \circ h$ is $p$-times continuously differentiable there. Since $y_{0} \in Y^{\prime}$ is arbitrary, $\alpha \circ h$ is $p$-times continuously differentiable on $Y^{\prime}$.

Let $x^{*} \in G^{*}$. By the preceding paragraph, $x^{*} \circ h$ is $p$-times continuously differentiable on $Y^{\prime}$. Consider a function $\alpha$ of the form $\beta \circ x^{*}$, where $\beta \in C^{p}(\mathbb{R})$. We have, for any $y \in Y^{\prime}$ and $w \in H$,

$$
D_{Y^{\prime}}^{p}(T(\alpha \cdot u))(y) w^{p}=\sum_{j=0}^{p}\left(\begin{array}{c}
p \\
j
\end{array}\right) D_{Y^{\prime}}^{j}\left(\beta \circ x^{*} \circ h\right)(y) w^{j} \cdot D_{Y^{\prime}}^{p-j} g(y)\left(w^{p-j}\right) .
$$


Applying the chain rule to $\beta \circ\left(x^{*} \circ h\right)$ gives, for $1 \leq j \leq p$,

$$
\begin{aligned}
D_{Y^{\prime}}^{j}\left(\beta \circ x^{*} \circ h\right)(y) w^{j}=\sum_{i=1}^{j} \sum_{\substack{k_{1}+\cdots+k_{i}=j \\
k_{1}, \ldots, k_{i} \geq 1}}\left(\begin{array}{c}
j \\
k_{1} k_{2} \cdots k_{i}
\end{array}\right) \frac{1}{i !} \\
\cdot \beta^{(i)}\left(\left(x^{*} \circ h\right)(y)\right) \cdot D_{Y^{\prime}}^{k_{1}}\left(x^{*} \circ h\right)(y) w^{k_{1}} \cdots D_{Y^{\prime}}^{k_{i}}\left(x^{*} \circ h\right)(y) w^{k_{i}} .
\end{aligned}
$$

Fix $y_{0} \in Y^{\prime}$ and let $x_{0}=h\left(y_{0}\right)$. Choose a sequence $\left(\beta_{m}\right)$ in $C^{p}(\mathbb{R})$ so that

(a) $\max _{0 \leq j \leq p} \sup _{m} \sup _{t \in \mathbb{R}}\left|\beta_{m}^{(j)}(t)\right|=M<\infty$,

(b) $\beta_{m}^{(p)}(t)=1$ if $1 / m \leq\left|t-x^{*}\left(x_{0}\right)\right| \leq 2$ and

(c) $\beta_{m}^{(j)}\left(x^{*}\left(x_{0}\right)\right)=0,0 \leq j \leq p$, for all $m$.

Let $f_{m}=\left(\beta_{m} \circ x^{*}\right) \cdot u$. Then $\left(f_{m}\right)$ is a bounded sequence in $C^{p}(X, E)$. Hence $\left(T f_{m}\right)$ is relatively weakly compact in $C^{p}(Y, F)$. Let $\left(y_{s}\right)$ be a sequence in $Y^{\prime}$ converging to $y_{0}$. The set $K=\left\{\left(y_{s}\right)\right\} \cup\left\{y_{0}\right\}$ is compact and thus the map $g \mapsto$ $D^{p} g_{\mid K}$ is a continuous linear operator from $C^{p}(Y, F)$ into $C\left(K, \mathcal{S}^{p}(H, F)\right)$; hence it is continuous with respect to the weak topologies on these spaces. Therefore, $\left(D^{p} T f_{m \mid K}\right)$ is relatively weakly compact in $C\left(K, \mathcal{S}^{p}(H, F)\right)$. To simplify notation, let us assume that $\left(D^{p} T f_{m \mid K}\right)$ converges weakly in $C\left(K, \mathcal{S}^{p}(H, F)\right)$. Then

$$
\lim _{s} \lim _{m} D^{p}\left(T f_{m}\right)\left(y_{s}\right)=\lim _{m} \lim _{s} D^{p}\left(T f_{m}\right)\left(y_{s}\right)=\lim _{m} D^{p}\left(T f_{m}\right)\left(y_{0}\right),
$$

where the limits are taken with respect to the weak topology on $\mathcal{S}^{p}(H, F)$. Of course, the same equation holds if the general derivative $D$ is replaced by the restricted derivative $D_{Y^{\prime}}$. By (c) and equations (6) and (7), $D_{Y^{\prime}}^{p}\left(T f_{m}\right)\left(y_{0}\right)=0$ for all $m$. Hence $\lim _{s} \lim _{m} D_{Y^{\prime}}^{p}\left(T f_{m}\right)\left(y_{s}\right)=0$ weakly. If $0 \leq i<p$, by (a) and (c),

$$
\begin{aligned}
\left|\beta_{m}^{(i)}\left(\left(x^{*} \circ h\right)\left(y_{s}\right)\right)\right| & =\left|\beta_{m}^{(i)}\left(\left(x^{*} \circ h\right)\left(y_{s}\right)\right)-\beta_{m}^{(i)}\left(\left(x^{*} \circ h\right)\left(y_{0}\right)\right)\right| \\
& \leq M\left|\left(x^{*} \circ h\right)\left(y_{s}\right)-\left(x^{*} \circ h\right)\left(y_{0}\right)\right| .
\end{aligned}
$$

Thus $\lim _{s} \sup _{m}\left|\beta_{m}^{(i)}\left(\left(x^{*} \circ h\right)\left(y_{s}\right)\right)\right|=0,0 \leq i<p$. From this and (7), it follows that $\lim _{s} \sup _{m}\left\|D_{Y^{\prime}}^{j}\left(\beta_{m} \circ x^{*} \circ h\right)\left(y_{s}\right)\right\|=0$ if $0 \leq j<p$, and that

$$
\begin{aligned}
\lim _{s} \sup _{m} \sup _{\|w\| \leq 1} \mid D_{Y^{\prime}}^{p}\left(\beta_{m} \circ x^{*} \circ h\right)\left(y_{s}\right) w^{p} & \\
& -\beta_{m}^{(p)}\left(\left(x^{*} \circ h\right)\left(y_{s}\right)\right)\left(D_{Y^{\prime}}\left(x^{*} \circ h\right)\left(y_{s}\right) w\right)^{p} \mid=0 .
\end{aligned}
$$

Therefore, from (6), we obtain for $w \in H$ and with limits taken in the weak topology on $F$,

$$
\begin{aligned}
0 & =\lim _{s} \lim _{m} D_{Y^{\prime}}^{p}\left(T f_{m}\right)\left(y_{s}\right) w^{p} \\
& =\lim _{s} \lim _{m} D_{Y^{\prime}}^{p}\left(\beta_{m} \circ x^{*} \circ h\right)\left(y_{s}\right) w^{p} \cdot g\left(y_{s}\right) \\
& =\lim _{s} \lim _{m} \beta_{m}^{(p)}\left(\left(x^{*} \circ h\right)\left(y_{s}\right)\right)\left(D_{Y^{\prime}}\left(x^{*} \circ h\right)\left(y_{s}\right) w\right)^{p} \cdot g\left(y_{s}\right) .
\end{aligned}
$$

Note that $\lim _{s} D_{Y^{\prime}}\left(x^{*} \circ h\right)\left(y_{s}\right) w=D_{Y^{\prime}}\left(x^{*} \circ h\right)\left(y_{0}\right) w$ and $\lim _{s} g\left(y_{s}\right)=g\left(y_{0}\right) \neq 0$. If $h\left(y_{s}\right) \neq x_{0}$, then $\lim _{m} \beta_{m}^{(p)}\left(\left(x^{*} \circ h\right)\left(y_{s}\right)\right)=1$ by $(\mathrm{b})$. Hence, if there is a sequence $\left(y_{s}\right)$ in $Y^{\prime}$ converging to $y_{0}$ so that $h\left(y_{s}\right) \neq x_{0}$ for all $s$, then $D_{Y^{\prime}}\left(x^{*} \circ h\right)\left(y_{0}\right)=0$. Otherwise, $h$ is constant on a neighborhood of $y_{0}$ in $Y^{\prime}$ and $D_{Y^{\prime}}\left(x^{*} \circ h\right)\left(y_{0}\right)=0$ also. 
Corollary 21. Suppose that $B\left(y_{0}, r\right) \subseteq Y$ and that $\Phi_{k}(y, \cdot)=0$ for all $y \in B\left(y_{0}, r\right)$ and $0<k \leq p$. Then $h$ is constant on $B\left(y_{0}, r\right)$.

Proof. Take $Y^{\prime}$ to be $B\left(y_{0}, r\right)$ in Proposition 20. For all $y \in B\left(y_{0}, r\right)$ and all $x^{*} \in G^{*}, D\left(x^{*} \circ h\right)(y)=0$. Thus, for all $x^{*} \in G^{*}, x^{*} \circ h$ is constant on $B\left(y_{0}, r\right)$. So $h$ is constant on $B\left(y_{0}, r\right)$.

Let $U$ be the subset of $Y$ consisting of all points $y \in Y$ so that $h$ is constant on a neighborhood of $y$. Then the set $Y^{\prime}=Y \backslash U$ is a closed (in $Y$ ) perfect subset of $Y$. Indeed, $U$ is obviously open and hence $Y^{\prime}$ is closed in $Y$. If $y_{0}$ is an isolated point in $Y^{\prime}$, then there exists $r>0$ so that $D h(y)=0$ for all $y \in B\left(y_{0}, r\right) \backslash\left\{y_{0}\right\}$. Thus $h$ is constant on $B\left(y_{0}, r\right)$ by continuity. This contradicts the fact that $y_{0} \notin U$.

Proposition 22. Suppose that $y_{0} \in Y^{\prime}$. Then $D\left(x^{*} \circ h\right)\left(y_{0}\right)=0$ for all $x^{*} \in G^{*}$.

Proof. By Lemma 19] $\Phi_{k}(y, \cdot)=0$ if $y \in Y^{\prime}$ and $0<k \leq p$. Fix $x^{*} \in G^{*}$. By Proposition 20, $D_{Y^{\prime}}\left(x^{*} \circ h\right)(y)=0$ for all $y \in Y^{\prime}$. In particular, if $\left(y_{n}\right)$ is a sequence in $Y^{\prime} \backslash\left\{y_{0}\right\}$ converging to $y_{0}$, then

$$
\lim _{n} \frac{\left|\left(x^{*} \circ h\right)\left(y_{n}\right)-\left(x^{*} \circ h\right)\left(y_{0}\right)\right|}{\left\|y_{n}-y_{0}\right\|}=0 .
$$

Now let $\left(y_{n}\right)$ be a sequence in $U$ converging to $y_{0}$. We may assume that all $y_{n}$ belong to an open ball centered at $y_{0}$ contained in $Y$. For each $n$, set $t_{n}=\sup \{0 \leq$ $\left.t<1:(1-t) y_{0}+t y_{n} \in Y^{\prime}\right\}$ and $z_{n}=\left(1-t_{n}\right) y_{0}+t_{n} y_{n}$. Since $Y^{\prime}$ is closed in $Y$, $z_{n} \in Y^{\prime}$. Also, the segment $\left(z_{n}, y_{n}\right]$ is contained in $U$ and hence $h$ is constant there. By continuity, $h\left(z_{n}\right)=h\left(y_{n}\right)$. Thus

$$
\lim _{\substack{n \\ z_{n} \neq y_{0}}} \frac{\left|\left(x^{*} \circ h\right)\left(y_{n}\right)-\left(x^{*} \circ h\right)\left(y_{0}\right)\right|}{\left\|y_{n}-y_{0}\right\|} \leq \lim _{\substack{n \\ z_{n} \neq y_{0}}} \frac{\left|\left(x^{*} \circ h\right)\left(z_{n}\right)-\left(x^{*} \circ h\right)\left(y_{0}\right)\right|}{\left\|z_{n}-y_{0}\right\|}=0
$$

since $D_{Y^{\prime}}\left(x^{*} \circ h\right)\left(y_{0}\right)=0$, while for those $n$ where $z_{n}=y_{0}, h\left(y_{n}\right)=h\left(y_{0}\right)$. This proves that $D\left(x^{*} \circ h\right)\left(y_{0}\right)=0$ for all $x^{*} \in G^{*}$.

Theorem 23. Let $1 \leq p<\infty$ and let $T: C^{p}(X, E) \rightarrow C^{p}(Y, F)$ be a weakly compact, separating, nowhere trivial linear operator. Then there is a partition of $Y$ into clopen subsets $\left(Y_{\alpha}\right)$ so that the support map is constant on each $Y_{\alpha}$.

Proof. Fix $x^{*} \in G^{*}$. If $y \in U$, then $h$ is constant on a neighborhood of $y$. Thus $D\left(x^{*} \circ h\right)(y)=0$. On the other hand, if $y \in Y^{\prime}$, then $D\left(x^{*} \circ h\right)(y)=0$ by Proposition 22. It follows that if a segment $\left[y_{1}, y_{2}\right]$ lies in $Y$, then $x^{*}\left(h\left(y_{1}\right)\right)=x^{*}\left(h\left(y_{2}\right)\right)$. Since this holds for all $x^{*} \in G^{*}, h\left(y_{1}\right)=h\left(y_{2}\right)$. Hence $h$ is constant on connected components of $Y$. As $Y$ is locally connected, the connected components of $Y$ are clopen in $Y$.

\section{COMPaCt AND WEAKly COMPACT MAPpings}

Proposition 24. Assume that $1 \leq p \leq \infty$ and $0 \leq q \leq \infty$. Let $T: C^{p}(X, E) \rightarrow$ $C^{q}(Y, F)$ be a nowhere trivial separating linear map. If $T$ maps an open set onto a bounded set, then the support map $h$ of $T$ maps $Y$ onto a relatively compact set in $X$. Moreover, in the representation of $T$ given by (2) in Theorem 10 , there exists $\ell \in \mathbb{N}_{p}$ such that $\Phi_{k}=0$ for all $k>\ell$. 
Proof. The map $T$ is continuous and hence we have the representation of $T$ given by Theorem 10. By the assumption, there exist $\ell \in \mathbb{N}_{p}$ and a compact set $L$ in $X$ so that the set $\left\{f: \rho_{L, \ell}(f)<1\right\}$ is mapped onto a bounded set. Suppose that there exists $y_{0} \in Y$ with $h\left(y_{0}\right) \notin L$. Choose $r>0$ so that $B\left(h\left(y_{0}\right), 2 r\right) \cap L=\emptyset$. Denoting by $\varphi$ the assumed bump function on $G$, for any $k \in \mathbb{N}_{p}$ and any $S \in$ $\mathcal{S}^{k}(G, E)$, the function $f \in C^{p}(X, E)$ given by $f(x)=\varphi\left(\frac{1}{r}\left(x-h\left(y_{0}\right)\right) S\left(x-h\left(y_{0}\right)\right)^{k}\right.$ is identically 0 on a neighborhood of $L$. Thus $\rho_{L, \ell}(m f)=0$ for any $m \in \mathbb{N}$. Hence the sequence $(m T f)_{m=1}^{\infty}$ is bounded in the topology on $C^{q}(Y, F)$. Therefore, $T f=0$. In particular, using the representation of $T, k ! \Phi_{k}\left(y_{0}, S\right)=T f\left(y_{0}\right)=0$. This shows that $\Phi_{k}\left(y_{0}, \cdot\right)=0$ for all $k \in \mathbb{N}_{p}$, contrary to the assumption that $T$ is nontrivial at the point $y_{0}$. We have shown that $h(Y)$ is contained in the compact set $L$, and hence is relatively compact.

If $p<\infty$, then the second assertion of the proposition is obvious, since we may take $\ell=p$. If $p=\infty$, take $\ell$ as in the previous paragraph. Let $y_{0} \in Y$. By the proof of Proposition[15, if $D^{j} f\left(h\left(y_{0}\right)\right)=0,0 \leq j \leq \ell$, then $D^{m}(T f)\left(y_{0}\right)=0, m \in \mathbb{N} \cup\{0\}$. In particular, $T f\left(y_{0}\right)=0$. Given $k>\ell$ and $S \in \mathcal{S}^{k}(G, E)$, consider the function $f(x)=S\left(x-h\left(y_{0}\right)\right)^{k}$. By the above, $T f\left(y_{0}\right)=0$. Thus $k ! \Phi_{k}\left(y_{0}, S\right)=0$. This proves that $\Phi_{k}=0$ for all $k>\ell$.

Corollary 13. Theorems 18 and 23 and Proposition 24 provide information on the support map of $T$ in various cases. We now consider how (weak) compactness of $T$ translates to the maps $\Phi_{k}$ in the representation of $T$. The key observation is given in the next proposition.

Proposition 25. Define the map $J: C^{q}(Y, F) \rightarrow \prod_{K, m} C\left(K, \mathcal{S}^{m}(H, F)\right)$ by $J f=$ $\left(D^{m} f_{\mid K}\right)_{K, m}$, where the product is taken over all compact subsets $K$ of $Y$ and all $m \in \mathbb{N}_{q}$. Then $J$ is a linear homeomorphic embedding with closed range. In particular, a subset $V$ of $C^{q}(Y, F)$ is relatively compact, respectively, relatively weakly compact, if and only if $J V$ is relatively compact, respectively, relatively weakly compact.

Proof. It is clear that $J$ is a linear homeomorphic embedding. If $J$ has closed (= weakly closed, because of convexity) range, then the final statement of the proposition follows easily.

Suppose that $\left(f_{\alpha}\right)$ is a net in $C^{q}(Y, F)$ so that $\left(J f_{\alpha}\right)$ converges. In particular, for each $y \in Y$, and each $m,\left(D^{m} f_{\alpha}(y)\right)_{\alpha}$ converges to some $g_{m}(y)$. We claim that the function $g_{m}: Y \rightarrow \mathcal{S}^{m}(H, F)$ is continuous. Indeed, let $\left(y_{k}\right)$ be a sequence in $Y$ converging to some $y_{0} \in Y$. Then $K=\left\{y_{k}: k \in \mathbb{N} \cup\{0\}\right\}$ is a compact subset of $Y$. Hence $\left(D^{m} f_{\alpha \mid K}\right)_{\alpha}$ converges in $C\left(K, \mathcal{S}^{m}(H, F)\right)$. The limit must be $g_{m \mid K}$. Thus, $g_{m \mid K}$ is continuous on $K$. In particular, $\lim _{k} g_{m}\left(y_{k}\right)=g_{m}\left(y_{0}\right)$. This proves that $g_{m}$ is continuous at any $y_{0} \in Y$. Next, we show that $D g_{m}=g_{m+1}$ if $0 \leq m<q$. Fix $y_{0} \in Y$ and let $V$ be a convex open neighborhood of $y_{0}$ in $Y$. For all $y \in V$ and all $\alpha$,

$$
D^{m} f_{\alpha}(y)-D^{m} f_{\alpha}\left(y_{0}\right)=\int_{0}^{1} D^{m+1} f_{\alpha}\left((1-t) y_{0}+t y\right)\left(y-y_{0}\right) d t .
$$

Since $\left(D^{m} f_{\alpha}\right)_{\alpha}$ and $\left(D^{m+1} f_{\alpha}\right)_{\alpha}$ converge uniformly on the segment $\left[y_{0}, y\right]$ to $g_{m}$ and $g_{m+1}$ respectively, we have

$$
g_{m}(y)-g_{m}\left(y_{0}\right)=\int_{0}^{1} g_{m+1}\left((1-t) y_{0}+t y\right)\left(y-y_{0}\right) d t .
$$


Hence

$$
\frac{\left\|g_{m}(y)-g_{m}\left(y_{0}\right)-g_{m+1}\left(y_{0}\right)\left(y-y_{0}\right)\right\|}{\left\|y-y_{0}\right\|} \leq \sup _{s \in\left[y_{0}, y\right]}\left\|g_{m+1}(s)-g_{m+1}\left(y_{0}\right)\right\| .
$$

Because of the continuity of $g_{m+1}$, we find that $D g_{m}\left(y_{0}\right)=g_{m+1}\left(y_{0}\right)$, as desired. It now follows easily that $D^{m} g_{0}=g_{m}$ and so $g_{0} \in C^{q}(Y, F)$. For each compact subset $K$ of $Y$ and each $m,\left(D^{m} f_{\alpha \mid K}\right)_{\alpha}$ converges in $C\left(K, \mathcal{S}^{m}(H, F)\right)$ by assumption, and the limit must be $g_{m \mid K}=D^{m} g_{0 \mid K}$. Therefore, $\left(J f_{\alpha}\right)_{\alpha}$ converges to $J g_{0}$. This proves that $J$ has closed range.

Theorem 26. Assume that $1 \leq p \leq q \leq \infty$. Let $T: C^{p}(X, E) \rightarrow C^{q}(Y, F)$ be a compact, separating, nowhere trivial linear operator with support map $h$. Then there exist $\ell \in \mathbb{N}_{p}$, functions $\Phi_{k}: Y \times \mathcal{S}^{k}(G, E) \rightarrow F, 0 \leq k \leq \ell$, a relatively compact set $\left(x_{\alpha}\right)$ in $X$ and a partition $\left(Y_{\alpha}\right)$ of $Y$ into clopen subsets of $Y$ so that

$$
T f(y)=\sum_{k=0}^{\ell} \Phi_{k}\left(y, D^{k} f\left(x_{\alpha}\right)\right)
$$

for all $f \in C^{p}(X, E)$ and all $y \in Y_{\alpha}$. For a given $y, \Phi_{k}(y, \cdot)$ is a bounded linear operator from $\mathcal{S}^{k}(G, E)$ into $F$. For each $S \in \mathcal{S}^{k}(G, E), \Phi_{k}(y, S)$ belongs to $C^{q}(Y, F)$ as a function of $y$. Moreover, for any $k \leq \ell$,

(a) for any $y \in Y$ and $m \in \mathbb{N}_{q}$, the $\operatorname{map} D_{y}^{m} \Phi_{k}(y, \cdot): \mathcal{S}^{k}(G, E) \rightarrow \mathcal{S}^{m}(H, F)$ is a compact operator $\left(D_{y}\right.$ denotes the derivative with respect to $\left.y\right)$;

(b) for each $m \in \mathbb{N}_{q}$, the map $y \mapsto D_{y}^{m} \Phi_{k}(y, \cdot)$ from $Y$ into the space of bounded linear operators $L\left(\mathcal{S}^{k}(G, E), \mathcal{S}^{m}(H, F)\right)$ is continuous with respect to the operator norm topology.

Conversely, any operator $T$ satisfying all of the above is a compact, separating linear operator from $C^{p}(X, E)$ into $C^{q}(Y, F)$.

Proof. Suppose that $T$ is a compact, separating, nowhere trivial linear operator. By Theorem 10, $T$ has the representation given by (2). By Corollary 13, Theorem 18 or Theorem 23, there is a partition $\left(Y_{\alpha}\right)$ of $Y$ into clopen subsets so that the support map $h$ is constant on each $Y_{\alpha}$. Denote the value of $h$ on $Y_{\alpha}$ by $x_{\alpha}$. By Proposition 24, the set $\left(x_{\alpha}\right)$ is relatively compact in $X$ and there exists $\ell \in \mathbb{N}_{p}$ such that $\Phi_{k}=0$ for all $k>\ell$. Hence we obtain the representation (8) of $T$. Given $k \leq \ell, S \in \mathcal{S}^{k}(G, E)$ and $\alpha$, let $f(x)=S\left(x-x_{\alpha}\right)^{k}$. Then $T f(y)=k ! \Phi_{k}(y, S)$ for all $y \in Y_{\alpha}$. Since $Y_{\alpha}$ is open, this proves that $\Phi_{k}(y, S)$ is $C^{q}$ as a function of $y \in Y_{\alpha}$. As this applies to all $\alpha, \Phi_{k}(y, S)$ belongs to $C^{q}(Y, F)$ as a function of $y$.

From compactness of $T$, we obtain $i \in \mathbb{N}_{p}$ and a compact set $L$ in $X$ so that $\left\{T f: \rho_{L, i}(f)<1\right\}$ is relatively compact in $C^{q}(Y, F)$. By Proposition 25. $\left\{D^{m}(T f)_{\mid K}: \rho_{L, i}(f)<1\right\}$ is relatively compact in $C\left(K, \mathcal{S}^{m}(H, F)\right)$ for each $m \in \mathbb{N}_{q}$ and each compact set $K$ in $Y$. By Arzelà-Ascoli's Theorem [7, Theorem IV.6.7], $\left\{D^{m}(T f)(y): \rho_{L, i}(f)<1\right\}$ is relatively compact in $\mathcal{S}^{m}(H, F)$ for each $y \in K$ and $\left\{D^{m}(T f)_{\mid K}: \rho_{L, i}(f)<1\right\}$ is equicontinuous on $K$. Given $k \leq \ell$ and $\alpha$, define $f_{S}(x)=S\left(x-x_{\alpha}\right)^{k}$ for all $S \in \mathcal{S}^{k}(G, E)$. Since $L$ is compact, there exists $\varepsilon>0$ so that $\rho_{L, i}\left(f_{S}\right)<1$ whenever $\|S\| \leq \varepsilon$. Let $m \in \mathbb{N}_{q}$. By direct computation, $D^{m}\left(T f_{S}\right)(y)=k ! D_{y}^{m} \Phi_{k}(y, S)$ for all $y \in Y_{\alpha}$. Choose $K$ to be any singleton $\{y\}$ in $Y_{\alpha}$. Then $\left\{k ! D_{y}^{m} \Phi_{k}(y, S):\|S\| \leq \varepsilon\right\}$ is relatively compact. Thus $D_{y}^{m} \Phi_{k}(y, \cdot)$ is a compact operator. On the other hand, choose $K$ to be any convergent sequence $\left(y_{j}\right)$ in $Y_{\alpha}$ together with the limit $y_{0}$. Since the set $\left\{D^{m}\left(T f_{S}\right):\|S\| \leq \varepsilon\right\}$ 
is equicontinuous on $K$,

$$
\lim _{j} \sup _{\|S\| \leq \varepsilon}\left\|D_{y}^{m} \Phi_{k}\left(y_{j}, S\right)-D_{y}^{m} \Phi_{k}\left(y_{0}, S\right)\right\|=0 .
$$

This proves that the function $y \mapsto D_{y}^{m} \Phi_{k}(y, \cdot)$ is continuous with respect to the norm topology on $L\left(\mathcal{S}^{k}(G, E), \mathcal{S}^{m}(H, F)\right)$.

In the converse direction, let $\ell$ be given by the statement of the theorem and $L$ be a compact set in $X$ containing $\left(x_{\alpha}\right)$. From conditions (a) and (b) and equation (8), one easily deduces that for all $m \in \mathbb{N}_{q}$ and all compact sets $K,\left\{D^{m}(T f)(y)\right.$ : $\left.\rho_{L, \ell}(f)<1\right\}$ is relatively compact in $\mathcal{S}^{m}(H, F)$ for each $y \in K$ and $\left\{D^{m}(T f)_{\mid K}\right.$ : $\left.\rho_{L, \ell}(f)<1\right\}$ is equicontinuous on $K$. Hence $\left\{D^{m}(T f)_{\mid K}: \rho_{L, \ell}(f)<1\right\}$ is relatively compact in $C\left(K, \mathcal{S}^{m}(H, F)\right)$ by Arzelà-Ascoli's Theorem. Relative compactness of the set $\left\{T f: \rho_{L, \ell}(f)<1\right\}$ follows from Proposition 25.

Given a bounded linear operator $T$ mapping between Banach spaces, let $T^{*}$ denote its adjoint. If $G_{1}$ and $G_{2}$ are Banach spaces, the strong operator topology on $L\left(G_{1}, G_{2}\right)$ is the topology generated by the seminorms $\rho_{u}(T)=\|T u\|, u \in G_{1}$.

Theorem 27. Assume that $1 \leq p \leq q \leq \infty$. Let $T: C^{p}(X, E) \rightarrow C^{q}(Y, F)$ be a weakly compact, separating, nowhere trivial linear operator with support map $h$. Then there exist $\ell \in \mathbb{N}_{p}$, functions $\Phi_{k}: Y \times \mathcal{S}^{k}(G, E) \rightarrow F, 0 \leq k \leq \ell$, a relatively compact set $\left(x_{\alpha}\right)$ in $X$ and a partition $\left(Y_{\alpha}\right)$ of $Y$ into clopen subsets of $Y$ so that

$$
T f(y)=\sum_{k=0}^{\ell} \Phi_{k}\left(y, D^{k} f\left(x_{\alpha}\right)\right)
$$

for all $f \in C^{p}(X, E)$ and all $y \in Y_{\alpha}$. For a given $y, \Phi_{k}(y, \cdot)$ is a bounded linear operator from $\mathcal{S}^{k}(G, E)$ into $F$. For each $S \in \mathcal{S}^{k}(G, E), \Phi_{k}(y, S)$ belongs to $C^{q}(Y, F)$ as a function of $y$. Moreover, for any $k \leq \ell$,

(c) for any $y \in Y$ and $m \in \mathbb{N}_{q}$, the map $D_{y}^{m} \Phi_{k}(y, \cdot): \mathcal{S}^{k}(G, E) \rightarrow \mathcal{S}^{m}(H, F)$ is a weakly compact operator;

(d) for each $m \in \mathbb{N}_{q}$, the map $y \mapsto\left(D_{y}^{m} \Phi_{k}(y, \cdot)\right)^{* *}$ from $Y$ into the space of bounded linear operators $L\left(\mathcal{S}^{k}(G, E)^{* *}, \mathcal{S}^{m}(H, F)^{* *}\right)$ is continuous with respect to the strong operator topology.

Conversely, any operator $T$ satisfying all of the above is a weakly compact, separating linear operator from $C^{p}(X, E)$ into $C^{q}(Y, F)$.

Proof. Following the proof of Theorem [26, it suffices to show that an operator $T$ of the form (8) is weakly compact if and only if conditions (c) and (d) hold. Let $J$ be the map defined in Proposition 25. By that result, a set $V$ is relatively weakly compact in $C^{q}(Y, F)$ if and only if $J V$ is relatively weakly compact in $\prod_{K, m} C\left(K, \mathcal{S}^{m}(H, F)\right)$. By [17, Theorem IV.4.3], the weak topology on $\prod_{K, m} C\left(K, \mathcal{S}^{m}(H, F)\right)$ is the product of the weak topologies on $C\left(K, \mathcal{S}^{m}(H, F)\right)$. Thus, by Tychonoff's Theorem, $V$ is relatively weakly compact if and only if $\left\{D^{m} f_{\mid K}: f \in V\right\}$ is relatively weakly compact in $C\left(K, \mathcal{S}^{m}(H, F)\right)$ for every $m \in \mathbb{N}_{q}$, and every compact subset $K$ of $Y$.

Assume that $T$ is weakly compact. There exist a compact subset $L$ of $X$ and $i \in \mathbb{N}_{p}$, so that $\left\{T f: \rho_{L, i}(f)<1\right\}$ is relatively weakly compact. Given $k \leq \ell$ and $\alpha$, define $f_{S}(x)=S\left(x-x_{\alpha}\right)^{k}$ for all $S \in \mathcal{S}^{k}(G, E)$. Since $L$ is compact, there exists 
$\varepsilon>0$ so that $\rho_{L, i}\left(f_{S}\right)<1$ whenever $\|S\| \leq \varepsilon$. Choose $K$ to be any singleton $\{y\}$ in $Y_{\alpha}$. Then for each $m \in \mathbb{N}_{q}$,

$$
\left\{D^{m}\left(T f_{S}\right)(y):\|S\| \leq \varepsilon\right\}=\left\{k ! D_{y}^{m} \Phi_{k}(y, S):\|S\| \leq \varepsilon\right\}
$$

is relatively weakly compact in $C\left(\{y\}, \mathcal{S}^{m}(H, F)\right)=\mathcal{S}^{m}(H, F)$. Hence condition (c) holds. On the other hand, choose $K$ to be any convergent sequence $\left(y_{j}\right)$ in $Y_{\alpha}$ together with the limit $y_{0}$. Let $m \in \mathbb{N}_{q}$ and denote by $B$ the closed unit ball of $\left(\mathcal{S}^{m}(H, F)\right)^{*}$, endowed with the weak ${ }^{*}$ topology, which renders it into a compact Hausdorff space. The map $I: C\left(K, \mathcal{S}^{m}(H, F)\right) \rightarrow C(K \times B)$ given by $I g(y, \gamma)=$ $\gamma(g(y))$ is a linear isometric embedding. Set $W=\left\{D^{m}\left(T f_{S}\right)_{\mid K}:\|S\| \leq \varepsilon\right\}$. Then $I W$ is relatively weakly compact in $C(K \times B)$. By [7, Theorem IV.6.14], the closure of $I W$ in the pointwise topology is contained in $C(K \times B)$. Denote by $R_{y}$ the operator $D_{y}^{m} \Phi_{k}(y, \cdot)$. Suppose that $S \in \mathcal{S}^{k}(G, E)^{* *}$ with $\|S\| \leq \varepsilon$. Define $g_{S}$ : $K \times B \rightarrow \mathbb{R}$ by $g_{S}(y, \gamma)=\left(R_{y}^{* *} S\right)(\gamma)$. There exists a net $\left(S_{\beta}\right)$ in $\mathcal{S}^{k}(G, E),\left\|S_{\beta}\right\| \leq \varepsilon$, that converges to $S$ in the weak* topology. Since $R_{y}\left(S_{\beta}\right)=D^{m}\left(T f_{S_{\beta} / k !}\right)(y)$, the functions $(y, \gamma) \mapsto \gamma\left(R_{y} S_{\beta}\right)$ belong to $I W \subseteq C(K \times B)$ and converge pointwise to $g_{S}$. Thus $g_{S}$ is continuous on $K \times B$. Therefore, $\lim _{j} g_{S}\left(y_{j}, \gamma\right)=g_{S}\left(y_{0}, \gamma\right)$ uniformly for $\gamma \in B$, which means that $\lim _{j} R_{y_{j}}^{* *} S=R_{y_{0}}^{* *} S$ in the norm of $\left(\mathcal{S}^{m}(H, F)\right)^{* *}$. This proves condition $(\mathrm{d})$.

In the converse direction, let $\ell$ be given by the statement of the theorem and $L$ be a compact set in $X$ containing $\left(x_{\alpha}\right)$. We claim that $\left\{T f: \rho_{L, \ell}(f)<1\right\}$ is relatively weakly compact in $C^{q}(Y, F)$. By the discussion in the first paragraph of the proof and using the isometric embedding $I$ as above, it suffices to show that for each $m \in \mathbb{N}_{q}$, and each compact subset $K$ of some $Y_{\alpha}$, the set $\left\{I D^{m}(T f)_{\mid K}: \rho_{L, \ell}(f)<1\right\}$ is relatively weakly compact in $C(K \times B)$. By [7, Theorem IV.6.14], we only need to show that the pointwise closure of this set is contained in $C(K \times B)$. Let $\left(f_{\beta}\right)$ be a net with $\rho_{L, \ell}\left(f_{\beta}\right)<1$ for all $\beta$ and assume that $\left(I D^{m}\left(T f_{\beta}\right)_{\mid K}\right)_{\beta}$ converges pointwise to a function $g$ on $K \times B$. For $0 \leq k \leq \ell,\left\|D^{k} f_{\beta}\left(x_{\alpha}\right)\right\|<1$. Thus, we may assume that $\left(D^{k} f_{\beta}\left(x_{\alpha}\right)\right)_{\beta}$ converges to some $S_{k} \in\left(\mathcal{S}^{k}(G, E)\right)^{* *}$ in the weak* topology. Denote by $R_{k, y}$ the operator $D_{y}^{m} \Phi_{k}(y, \cdot)$. By condition (c), $R_{k, y}$ is a weakly compact operator for each $y$. Hence $R_{k, y}^{* *} S_{k} \in \mathcal{S}^{m}(H, F)$ and $\lim _{\beta} R_{k, y}\left(D^{k} f_{\beta}\left(x_{\alpha}\right)\right)=R_{k, y}^{* *}\left(S_{k}\right)$ weakly. Therefore, for all $y \in K$ and all $\gamma \in B$,

$$
\begin{aligned}
g(y, \gamma) & =\lim _{\beta} \gamma\left(D^{m}\left(T f_{\beta}\right)(y)\right)=\lim _{\beta} \sum_{k=0}^{\ell} \gamma\left(R_{k, y}\left(D^{k} f_{\beta}\left(x_{\alpha}\right)\right)\right) \\
& =\sum_{k=0}^{\ell} \gamma\left(R_{k, y}^{* *}\left(S_{k}\right)\right) .
\end{aligned}
$$

It follows that, for each $y \in K, g(y, \cdot)$ is a continuous function on $B$. By condition (d), if $\left(y_{j}\right)$ converges to $y$ in $K$, then $\left(\gamma\left(R_{k, y_{j}}^{* *} S_{k}\right)\right)_{j}$ converges to $\gamma\left(R_{k, y}^{* *} S_{k}\right)$ uniformly in $\gamma \in B$; that is, $g\left(y_{j}, \gamma\right)$ converges to $g(y, \gamma)$ uniformly in $\gamma \in B$. Since, by the above, each $g\left(y_{j}, \cdot\right)$ is a continuous function on $B$, it follows easily that $g$ is a continuous function on $K \times B$.

\section{THE CASE $p>q$}

The example at the end of Section 4 tells us that when $p>q$, the support map of a compact, disjointness preserving linear operator $T: C^{p}(X, E) \rightarrow C^{q}(Y, F)$ need 
not be degenerate. Indeed, we shall see that the support map need not even be differentiable at all points of $Y$. In this section, we will present the counterparts of Theorems 26 and 27 when $p>q$ and $X$ is an open set in $\mathbb{R}^{n}$.

As indicated, in this section, $X$ will be an open set in $\mathbb{R}^{n}$. A multiindex is an $n$-tuple of nonnegative integers $\lambda=\left(\lambda_{1}, \ldots, \lambda_{n}\right)$. The order of $\lambda$ is $|\lambda|=\sum_{i=1}^{n} \lambda_{i}$. If $x=\left(x_{1}, \ldots, x_{n}\right) \in \mathbb{R}^{n}$ and $\lambda$ is a multiindex, let $x^{\lambda}=\prod_{i=1}^{n} x^{\lambda_{i}}$. Given a multiindex $\lambda$ and $u \in E$, let $S_{\lambda, u}$ be the operator in $\mathcal{S}^{k}\left(\mathbb{R}^{n}, E\right), k=|\lambda|$, so that $S_{\lambda, u} x^{k}=x^{\lambda} u$. The set of operators $\left\{S_{\lambda, u}:|\lambda|=k, u \in E\right\}$ span $\mathcal{S}^{k}\left(\mathbb{R}^{n}, E\right)$. For the rest of the section, let $T: C^{p}(X, E) \rightarrow C^{q}(Y, F)$ be given a continuous, separating, nowhere trivial linear operator. Denote by $h$ the support map. By Theorem 10, $T$ has the representation

$$
T f(y)=\sum_{k} \Phi_{k}\left(y, D^{k} f(h(y))\right),
$$

where the sum is locally finite.

Proposition 28. Suppose that $1 \leq i \leq n$. There is a dense open subset $U_{i}$ of $Y$ so that for all $y_{0} \in U_{i}$, there exist $m_{0} \geq 0$ and an open neighborhood $V$ of $y_{0}$ so that $\Phi_{|\lambda|}\left(y, S_{\lambda, u}\right)=0$ if $y \in V, u \in E$ and $\lambda_{i}>m_{0}$, and $\Phi_{|\mu|}\left(y_{0}, S_{\mu, u}\right) \neq 0$ for some multiindex $\mu$ with $\mu_{i}=m_{0}$ and some $u \in E$.

Proof. $Y$ can be covered by open subsets $W$, where for each $W$, there exists $k_{0}=$ $k_{0}(W) \in \mathbb{N}$ such that $\Phi_{k}(y, \cdot)=0$ for all $k>k_{0}$ and all $y \in W$. For $m \geq 0$, let

$$
\begin{aligned}
& A_{m}=\left\{y \in Y: \text { there exist } \lambda \text { and } u \text { so that } \lambda_{i}=m, \Phi_{|\lambda|}\left(y, S_{\lambda, u}\right) \neq 0\right\}, \\
& B_{m}=\left\{y \in Y: \text { for all } \lambda \text { and } u \text { with } \lambda_{i}=m, \Phi_{|\lambda|}\left(y, S_{\lambda, u}\right)=0\right\} .
\end{aligned}
$$

Clearly, $A_{m}$ is open and $A_{m} \cup B_{m}=Y$. For each $W$ and $k_{0}$ as above,

$$
U_{W}=W \cap\left[A_{k_{0}} \cup \bigcup_{m=0}^{k_{0}-1}\left(A_{m} \cap \operatorname{int} B_{m+1} \cap \cdots \cap \operatorname{int} B_{k_{0}}\right) \cup \bigcap_{m=0}^{k_{0}} \operatorname{int} B_{m}\right]
$$

is a dense open set in $W$. If $y \in W \cap \bigcap_{m=0}^{k_{0}}$ int $B_{m}$, then $\Phi_{|\lambda|}\left(y, S_{\lambda, u}\right)=0$ whenever $\lambda_{i} \leq k_{0}$ and $u \in E$. Thus $\Phi_{k}(y, \cdot)=0$ for all $k \leq k_{0}$. Since $y \in W, \Phi_{k}(y, \cdot)=0$ for $k>k_{0}$ as well. This contradicts the fact that $T$ is nontrivial at $y$. Hence

$$
U_{W}=W \cap\left[A_{k_{0}} \cup \bigcup_{m=0}^{k_{0}-1}\left(A_{m} \cap \operatorname{int} B_{m+1} \cap \cdots \cap \operatorname{int} B_{k_{0}}\right)\right] .
$$

The set $U_{i}=\bigcup_{W} U_{W}$ fulfills the conditions of the proposition.

Let $U$ be the set $\bigcap_{i=1}^{n} U_{i}$. Then $U$ is a dense open subset of $Y$. Denote the $i$-th component of $h$ by $h_{i}$. Given multiindices $\lambda=\left(\lambda_{1}, \ldots, \lambda_{n}\right)$ and $\nu=\left(\nu_{1}, \ldots, \nu_{n}\right)$, we write $\lambda \leq \nu$ to mean that $\lambda_{i} \leq \nu_{i}, 1 \leq i \leq n$. In this case, we let the binomial coefficient $\left(\begin{array}{c}\nu \\ \lambda\end{array}\right)$ be $\prod_{i=1}^{n}\left(\begin{array}{c}\nu_{i} \\ \lambda_{i}\end{array}\right)$.

Proposition 29. For $1 \leq i \leq n, h_{i}$ is $C^{q}$ on the set $U_{i}$. Therefore, $h$ is $C^{q}$ on the dense open set $U$ of $Y$.

Proof. Take $y_{0} \in U_{i}$. We may assume that $i=1$ and $h\left(y_{0}\right)=0$. Let $V$ and $m_{0}$ be given by Proposition 28 and let $\mu=\left(\mu_{1}, \ldots, \mu_{n}\right), u \in E$ and $v^{*} \in F^{*}$ be such that $\mu_{1}=m_{0}$ and $v^{*}\left(\Phi_{|\mu|}\left(y_{0}, S_{\mu, u}\right)\right) \neq 0$. If $0 \leq j \leq m_{0}+1$, let $f_{j}: X \rightarrow E$ 
be the function $f_{j}(x)=x^{\nu_{j}} u$, where $\nu_{j}$ is the multiindex $\left(j, \mu_{2}, \ldots, \mu_{n}\right)$. Then $f_{j} \in C^{p}(X, E)$ and, for all $y \in V$,

$$
T f_{j}(y)=\sum_{k=0}^{j \wedge m_{0}} \sum_{\substack{\lambda \leq \nu_{j} \\
\lambda_{1}=k}}|\lambda| !\left(\begin{array}{c}
\nu_{j} \\
\lambda
\end{array}\right) h(y)^{\nu_{j}-\lambda} \cdot \Phi_{|\lambda|}\left(y, S_{\lambda, u}\right) .
$$

If $0 \leq j \leq m_{0}$, set

$$
g_{j}(y)=\sum_{\substack{\lambda \leq \nu_{j} \\
\lambda_{1}=j}}|\lambda| !\left(\begin{array}{c}
\nu_{j} \\
\lambda
\end{array}\right) h(y)^{\nu_{j}-\lambda} \cdot v^{*}\left(\Phi_{|\lambda|}\left(y, S_{\lambda, u}\right)\right) .
$$

Then

$$
\begin{array}{r}
\sum_{k=0}^{j}\left(\begin{array}{l}
j \\
k
\end{array}\right) g_{j-k} h_{1}^{k}=v^{*} \circ T f_{j}, 0 \leq j \leq m_{0}, \text { and } \\
\sum_{k=1}^{m_{0}+1}\left(\begin{array}{c}
m_{0}+1 \\
k
\end{array}\right) g_{m_{0}+1-k} h_{1}^{k}=v^{*} \circ T f_{m_{0}+1}
\end{array}
$$

are $C^{q}$ functions on $V$. Consider functions $F_{j}: \mathbb{R}^{m_{0}+2} \rightarrow \mathbb{R}$ given by

$$
F_{j}\left(t_{0}, \ldots, t_{m_{0}}, s\right)=\sum_{k=0}^{j}\left(\begin{array}{l}
j \\
k
\end{array}\right) t_{j-k} s^{k}
$$

for $0 \leq j \leq m_{0}$ and $F_{m_{0}+1}\left(t_{0}, \ldots, t_{m_{0}}, s\right)=\sum_{k=1}^{m_{0}+1}\left(\begin{array}{c}m_{0}+1 \\ k\end{array}\right) t_{m_{0}+1-k} s^{k}$. Now

$$
\begin{aligned}
\sum_{j=0}^{m_{0}+1}(-1)^{j} & \left(\begin{array}{c}
m_{0}+1 \\
j
\end{array}\right) s^{m_{0}+1-j} \partial_{t_{i}} F_{j} \\
& =(-1)^{i} s^{m_{0}+1-i}\left(\begin{array}{c}
m_{0}+1 \\
i
\end{array}\right) \sum_{j=0}^{m_{0}+1-i}\left(\begin{array}{c}
m_{0}+1-i \\
j
\end{array}\right)(-1)^{j} \\
& =(-1)^{i} s^{m_{0}+1}\left(\begin{array}{c}
m_{0}+1 \\
i
\end{array}\right)(1-1)^{m_{0}+1-i}=0
\end{aligned}
$$

and

$$
\begin{aligned}
\sum_{j=0}^{m_{0}+1}(-1)^{j}\left(\begin{array}{c}
m_{0}+1 \\
j
\end{array}\right) s^{m_{0}+1-j} \partial_{s} F_{j} & \\
& =\sum_{j=1}^{m_{0}+1} \sum_{k=1}^{j}(-1)^{j}\left(\begin{array}{c}
m_{0}+1 \\
j
\end{array}\right)\left(\begin{array}{l}
j \\
k
\end{array}\right) k t_{j-k} s^{m_{0}+k-j},
\end{aligned}
$$

which yields, upon substituting $i=j-k$ and switching the order of summation,

$$
\begin{aligned}
& =\sum_{i=0}^{m_{0}}(-1)^{i} t_{i} s^{m_{0}-i}\left(\begin{array}{c}
m_{0}+1 \\
i
\end{array}\right)\left[\sum_{j=1}^{m_{0}+1-i}\left(\begin{array}{c}
m_{0}-i+1 \\
j
\end{array}\right) j(-1)^{j}\right] \\
& =\sum_{i=0}^{m_{0}}(-1)^{i} t_{i} s^{m_{0}-i}\left(\begin{array}{c}
m_{0}+1 \\
i
\end{array}\right)\left[\frac{d}{d t}(1-t)^{m_{0}-i+1}\right]_{\mid t=1} \\
& =(-1)^{m_{0}}\left(m_{0}+1\right) t_{m_{0}} .
\end{aligned}
$$


It follows that the function $F=\left(F_{0}, \ldots, F_{m_{0}+1}\right)$ has a local $C^{\infty}$ inverse at any $\left(t_{0}, \ldots, t_{m_{0}}, s\right)$ with $t_{m_{0}} \neq 0$. Since $F\left(g_{0}, \ldots, g_{m_{0}}, h_{1}\right)$ is $C^{q}$ on $V$ and, because $h\left(y_{0}\right)=0$,

$$
g_{m_{0}}\left(y_{0}\right)=|\mu| ! v^{*}\left(\Phi_{|\mu|}\left(y_{0}, S_{\mu, u}\right)\right) \neq 0,
$$

the function $h_{1}$, in particular, must be $C^{q}$ on a neighborhood of $y_{0}$.

The next example shows that $h$ need not be differentiable at all points of $Y$.

Example. Assume that $0 \leq q<p<\infty$. If $f \in C^{p}(\mathbb{R})$, then it is easy to check that the function

$$
g(t)= \begin{cases}\sum_{i=0}^{p} \frac{t^{i}}{i !} f^{(i)}(0) & t<0, \\ f(t) & t \geq 0\end{cases}
$$

belongs to $C^{p}(\mathbb{R})$. Consider the map $R: f \mapsto g$ as an operator from $C^{p}(\mathbb{R})$ to $C^{q}(I)$, where $I=(-1,1)$. Then $R$ is a continuous, separating, nowhere trivial linear operator. It is even compact as it maps the open set $\left\{f \in C^{p}: \max _{k \leq p} \sup _{|t| \leq 1}\left|f^{(k)}(t)\right|<\right.$ $1\}$ onto a compact subset of $C^{q}(I)$. However, the support map $h: \bar{I} \rightarrow \mathbb{R}$ of $R$, given by $h(t)=0$ if $t<0$ and $h(t)=t$ if $t \geq 0$, is not differentiable at 0 .

To proceed, we require a result from [15].

Proposition 30. 15, Proposition 8] Let $\mathfrak{X}$ be a Banach space and suppose that $\Phi: U \times \mathfrak{X} \rightarrow F$ has the following properties.

(a) For each $u \in \mathfrak{X}, \Phi(\cdot, u): U \rightarrow F$ belongs to $C^{q}(U, F)$. Denote the $j$-th derivative of this function by $D_{y}^{j} \Phi(\cdot, u)$.

(b) For each $y \in U$ and each $j \in \mathbb{N}_{q}, D_{y}^{j} \Phi(y, \cdot): \mathfrak{X} \rightarrow \mathcal{S}^{j}(H, F)$ is a bounded linear operator.

(c) For each $y_{0} \in U$ and each $j \in \mathbb{N}_{q}$, there exists $\varepsilon>0$ so that

$$
\sup _{y \in B\left(y_{0}, \varepsilon\right)} \sup _{\|u\| \leq 1}\left\|D_{y}^{j} \Phi(y, u)\right\|<\infty .
$$

If $f: U \rightarrow \mathfrak{X}$ is a $C^{q}$ function on $U$, then $\Psi: U \rightarrow F$ defined by $\Psi(y)=\Phi(y, f(y))$ belongs to $C^{q}(U, F)$. For each $m \in \mathbb{N}_{q}$ and each $y \in U$,

$$
D^{m} \Psi(y) s^{m}=\sum_{j=0}^{m}\left(\begin{array}{c}
m \\
j
\end{array}\right) D_{y}^{j} \Phi\left(y, D^{m-j} f(y) s^{m-j}\right) s^{j}, \quad s \in H .
$$

Applying Proposition 30 to the operator $T$ with the representation given at the beginning of the section, we obtain

Proposition 31. For any $k$ and any $S \in \mathcal{S}^{k}\left(\mathbb{R}^{n}, E\right)$, the function $y \mapsto \Phi_{k}(y, S)$ belongs to $C^{q}(U, F)$. Given $m \in \mathbb{N}_{q}$, denote the $m$-th derivative of this function by $D_{y}^{m} \Phi_{k}(\cdot, S)$. For each $y \in U$, the map $S \mapsto D_{y}^{m} \Phi_{k}(y, S)$ is a bounded linear operator from $\mathcal{S}^{k}\left(\mathbb{R}^{n}, E\right)$ to $\mathcal{S}^{m}(H, F)$. The set of all such operators is locally bounded on $U$; that is, for each $y_{0} \in U$, there exists $\varepsilon>0$ so that

$$
\sup _{y \in B\left(y_{0}, \varepsilon\right)} \sup _{\|S\| \leq 1}\left\|D_{y}^{m} \Phi_{k}(y, S)\right\|<\infty .
$$

Proof. We proceed by induction on $k$. Assume that the proposition holds up to and including $k-1$. (If $k=0$, the assumption is vacuously true.) If $S \in \mathcal{S}^{k}\left(\mathbb{R}^{n}, E\right)$, let $f_{S}: X \rightarrow E$ be the function $f_{S}(x)=S x^{k}$. Then

$$
T f_{S}(y)=\Phi_{k}(y, S)+\sum_{i=0}^{k-1} \frac{k !}{(k-i) !} \Phi_{i}\left(y, S h(y)^{k-i}\right) .
$$


Since $h$ is $C^{q}$ on $U$ by Proposition 29, it follows from the inductive hypothesis and Proposition 30 that the summation term above is a $C^{q}$ function of $y$ on $U$. Since $T f_{S} \in C^{q}(Y, F)$ as well, $\Phi_{k}(y, S)$ is a $C^{q}$ function of $y$ on $U$. Fix $y \in U$. By the continuity of $T$, for each $m$ in $\mathbb{N}_{q}$ and each $y \in Y, D^{m}\left(T f_{S}\right)(y)$ is a bounded linear operator of the variable $S$. Furthermore, by the inductive hypothesis and the differentiation formula (9), the $m$-th derivative with respect to $y$ of the summation term in (10) is a bounded linear operator of $S$. Thus the map $S \mapsto D_{y}^{m} \Phi_{k}(y, S)$ is a bounded linear operator. To obtain the final conclusion of the proposition, it suffices to show, in view of (9), (10) and the inductive hypothesis, that for any $y_{0} \in U$, there exists $\varepsilon>0$ so that

$$
\sup _{y \in B\left(y_{0}, \varepsilon\right)} \sup _{\|S\| \leq 1}\left\|D^{m}\left(T f_{S}\right)(y)\right\|<\infty .
$$

For each $y \in Y$, we have already observed that the linear operator $R_{m, y}: \mathcal{S}^{k}\left(\mathbb{R}^{n}, E\right)$ $\rightarrow \mathcal{S}^{m}(H, F), R_{m, y}(S)=D^{m}\left(T f_{S}\right)(y)$, is bounded. If $\left(y_{j}\right)$ converges to $y_{0}$, then

$$
R_{m, y_{j}}(S)=D^{m}\left(T f_{S}\right)\left(y_{j}\right) \rightarrow D^{m}\left(T f_{S}\right)\left(y_{0}\right)=R_{m, y_{0}}(S)
$$

since $T f_{S}$ is a $C^{q}$ function. By the uniform boundedness principle, the sequence of operators $\left(R_{m, y_{j}}\right)$ is uniformly bounded. Hence the operators $\left(R_{m, y}\right)_{y \in Y}$ are locally bounded (in the operator norm) on $Y$, yielding the desired result.

Now suppose that $T$ is also compact. There exist a compact set $L$ in $X$ and $\ell \in \mathbb{N}_{p}$ so that $\left\{T f: \rho_{L, \ell}(f)<1\right\}$ is relatively compact in $C^{q}(Y, F)$. For $k \in \mathbb{N}_{p}$ and $S \in \mathcal{S}^{k}\left(\mathbb{R}^{n}, E\right)$, let $f_{S}(x)=S x^{k}$. Then $\left\{T f_{S}:\|S\| \leq 1\right\}$ is relatively compact in $C^{q}(Y, F)$. Let $K=\left\{\left(y_{i}\right)\right\} \cup\left\{y_{0}\right\}$, where $\left(y_{i}\right)$ is a sequence in $Y$ converging to $y_{0} \in Y$. For each $m \in \mathbb{N}_{q},\left\{D^{m}\left(T f_{S}\right)_{\mid K}:\|S\| \leq 1\right\}$ is relatively compact in $C\left(K, \mathcal{S}^{m}(H, F)\right)$. Let $R_{m, y}(S)=D^{m}\left(T f_{S}\right)(y)$. Thus $\left\{R_{m, y}(S):\|S\| \leq 1\right\}$ is relatively compact for each $y \in K$ and the set of maps $y \mapsto R_{m, y}(S),\|S\| \leq 1$, is equicontinuous on $K$. Hence $R_{m, y}$ is a compact operator for any $y \in Y$ (since $K$ can be chosen to contain $y)$ and $\lim _{i} R_{m, y_{i}}=R_{m, y_{0}}$ in the operator norm of $L\left(\mathcal{S}^{k}\left(\mathbb{R}^{n}, E\right), \mathcal{S}^{m}(H, F)\right)$. Since $Y$ is metric, this means that $y \mapsto R_{m, y}$ is continuous with respect to the operator norm topology.

Theorem 32. Let $X$ be an open set in $\mathbb{R}^{n}$ and assume that $0 \leq q<p \leq \infty$. Suppose that $T: C^{p}(X, E) \rightarrow C^{q}(Y, F)$ is a compact, separating, nowhere trivial linear operator with support map $h$. Then there exist $\ell \in \mathbb{N}_{p}$ and functions $\Phi_{k}$ : $Y \times \mathcal{S}^{k}\left(\mathbb{R}^{n}, E\right) \rightarrow F, 0 \leq k \leq \ell$, so that $T$ has the representation

$$
T f(y)=\sum_{k=0}^{\ell} \Phi_{k}\left(y, D^{k} f(h(y))\right)
$$

for all $f \in C^{p}(X, E)$ and all $y \in Y$. For a given $y, \Phi_{k}(y, \cdot)$ is a bounded linear operator from $\mathcal{S}^{k}\left(\mathbb{R}^{n}, E\right)$ into $F$. The set $h(Y)$ is relatively compact in $X$. There exists a dense open set $U$ in $Y$ so that $h$ is $C^{q}$ on $U$. For each $S \in \mathcal{S}^{k}\left(\mathbb{R}^{n}, E\right)$, $\Phi_{k}(y, S)$ belongs to $C^{q}(U, F)$ as a function of $y \in U$. Moreover, for any $k \leq \ell$,

(a) for any $y \in U$ and $m \in \mathbb{N}_{q}$, the map $D_{y}^{m} \Phi_{k}(y, \cdot): \mathcal{S}^{k}\left(\mathbb{R}^{n}, E\right) \rightarrow \mathcal{S}^{m}(H, F)$ is a compact operator;

(b) for each $m \in \mathbb{N}_{q}$, the map $y \mapsto D_{y}^{m} \Phi_{k}(y, \cdot)$ from $U$ into the space of bounded linear operators $L\left(\mathcal{S}^{k}\left(\mathbb{R}^{n}, E\right), \mathcal{S}^{m}(H, F)\right)$ is continuous with respect to the operator norm topology. 
Proof. By Proposition 24, $T$ has the given representation for some $\ell \in \mathbb{N}_{p}$ and $h(Y)$ is relatively compact in $X$. By Theorem 10, $\Phi_{k}(y, \cdot): \mathcal{S}^{k}\left(\mathbb{R}^{n}, E\right) \rightarrow F$ is a bounded linear operator for each $y \in Y$. By Proposition 29, $h$ is $C^{q}$ on a dense open subset $U$ of $Y$. By Proposition [31, for each $S \in \mathcal{S}^{k}\left(\mathbb{R}^{n}, E\right), \Phi_{k}(y, S)$ belongs to $C^{q}(U, F)$ as a function of $y \in U$. Finally, using the notation above, rewrite equation (10) as

$$
\Phi_{k}(y, S)=\sum_{i=0}^{k-1} \frac{k !}{(k-i) !} \Phi_{i}\left(y, S h(y)^{k-i}\right)-T f_{S}(y) .
$$

For each $m \in \mathbb{N}_{q}$, observe that $D^{m}\left(T f_{S}\right)(y)=R_{m, y}(S)$. By the discussion preceding the theorem, $R_{m, y}$ is a compact operator and a continuous function of $y$ with respect to the operator norm topology. Differentiate the summation term $(m$ times) using (9) for $y \in U$. Assuming conditions (a) and (b) for $\Phi_{i}, 0 \leq i<k$, the $m$-th derivative with respect to $y$ of the summation term above is a compact operator of $S$, and is a continuous function of $y \in U$ with respect to the operator norm topology. Therefore, we obtain conditions (a) and (b) for $\Phi_{k}$.

Remarks. (a) If $\Phi_{k}\left(y_{0}, \cdot\right) \neq 0$ for some $k \in \mathbb{N}_{p}$ with $k+q>p$, then $h$ is constant on a neighborhood of $y_{0}$ by Theorem 12 .

(b) If, in addition to the conditions on $\Phi_{k}$ 's enunciated in the theorem, it is also assumed that for all $m \in \mathbb{N}_{q}$ and $1 \leq k \leq \ell$, the $m$-th derivative of

$$
\sum_{i=0}^{k-1} \frac{k !}{(k-i) !} \Phi_{i}\left(y, S h(y)^{k-i}\right)
$$

with respect to $y$, considered as a linear operator of the variable $S$, extends continuously in the operator norm topology to a function on $Y$, then the operator $T$ with the given representation is compact.

A similar result holds for weakly compact operators. The argument is similar to that of Theorem 32, but using the consideration for weak compactness as in the proof of Theorem 27. The details are omitted.

Theorem 33. Let $X$ be an open set in $\mathbb{R}^{n}$ and assume that $0 \leq q<p \leq \infty$. Suppose that $T: C^{p}(X, E) \rightarrow C^{q}(Y, F)$ is a weakly compact, separating, nowhere trivial linear operator with support map $h$. Then there exist $\ell \in \mathbb{N}_{p}$ and functions $\Phi_{k}: Y \times \mathcal{S}^{k}\left(\mathbb{R}^{n}, E\right) \rightarrow F, 0 \leq k \leq \ell$, so that $T$ has the representation

$$
T f(y)=\sum_{k=0}^{\ell} \Phi_{k}\left(y, D^{k} f(h(y))\right)
$$

for all $f \in C^{p}(X, E)$ and all $y \in Y$. For a given $y, \Phi_{k}(y, \cdot)$ is a bounded linear operator from $\mathcal{S}^{k}\left(\mathbb{R}^{n}, E\right)$ into $F$. The set $h(Y)$ is relatively compact in $X$. There exists a dense open set $U$ in $Y$ so that $h$ is $C^{q}$ on $U$. For each $S \in \mathcal{S}^{k}\left(\mathbb{R}^{n}, E\right)$, $\Phi_{k}(y, S)$ belongs to $C^{q}(U, F)$ as a function of $y \in U$. Moreover, for any $k \leq \ell$,

(a) for any $y \in U$ and $m \in \mathbb{N}_{q}$, the map $D_{y}^{m} \Phi_{k}(y, \cdot): \mathcal{S}^{k}\left(\mathbb{R}^{n}, E\right) \rightarrow \mathcal{S}^{m}(H, F)$ is a weakly compact operator;

(b) for each $m \in \mathbb{N}_{q}$, the map $y \mapsto\left(D_{y}^{m} \Phi_{k}(y, \cdot)\right)^{* *}$ from $U$ into the space of bounded linear operators $L\left(\mathcal{S}^{k}\left(\mathbb{R}^{n}, E\right)^{* *}, \mathcal{S}^{m}(H, F)^{* *}\right)$ is continuous with respect to the strong operator topology. 


\section{REFERENCES}

[1] J. Araujo, Realcompactness and spaces of vector-valued continuous functions, Fund. Math. 172 (2002), 27-40. MR1898401 (2003h:54033)

[2] J. Araujo, Linear biseparating maps between spaces of vector-valued differentiable functions and automatic continuity, Adv. Math. 187 (2004), 488-520. MR2078345 (2005c:47040)

[3] J. Araujo, E. Beckenstein and L. Narici, Biseparating maps and homeomorphic realcompactifications, J. Math. Anal. Appl. 192 (1995), 258-265. MR.1329423 (96b:46038)

[4] R.M. Aron, J. Gomez And J. Llavona, Homomorphisms between algebras of differentiable functions in infinite dimensions, Michigan Math. J. 35 (1988), 163-178. MR959264 (89j:46048)

[5] E. Beckenstein, L. Narici And A. Todd, Automatic continuity of linear maps on spaces of continuous functions, Manuscripta Math. 62 (1988), 257-275. MR966626 (89j:47020)

[6] R. Deville, G. Godefroy and Václav Zizler, Smoothness and renormings in Banach spaces, Pitman Monographs and Surveys in Pure and Applied Mathematics 64, 1993. MR.1211634(94d:46012)

[7] N. Dunford and J.T. Schwartz, Linear Operators, Part I, Wiley, New York. MR,1009162 (90g:47001a)

[8] L. Gillman and M. Jerison. Rings of Continuous Functions, D. van Nostrand, Princeton, 1960. MR0116199(22:6994)

[9] M. GonzÁlez AND J.M. Gutiérrez, Compact and weakly compact homomorphisms between algebras of differentiable functions, Proc. Amer. Math. Soc. 115 (1992), 1025-1029. MR.1087463 (92j:46095)

[10] J.M. GutiÉRrez AND J. Llavona, Composition operators between algebras of differentiable functions. Trans. Amer. Math. Soc. 338 (1993), 769-782. MR.1116313 (93j:46048)

[11] S. Hernandez, E. Beckenstein and L. Narici, Banach-Stone theorems and separating maps, Manuscripta Math. 86 (1995), 409-416. MR1324679 (95m:46054)

[12] K. Jarosz, Automatic continuity of separating linear isomorphisms, Canad. Math. Bull. 33 (1990), 139-144. MR1060366 (92j:46049)

[13] J.-S. JEANG AND Y.-F. LiN, Characterizations of disjointness preserving operators on vector-valued function spaces, Proc. Amer. Math. Soc. 136 (2008), 947-954. MR2361868 (2009a:47036)

[14] S. LANG, Differential and Riemannian Manifolds, Graduate Texts in Mathematics 160, Springer-Verlag, New York, 1995. MR1335233 (96d:53001)

[15] D.H. Leung and Y.-S. Wang, Local operators on $C^{p}$, J. Math. Anal. Appl. 381 (2011), 308-314. MR2796211

[16] Y.-F. Lin AND N.-C. Wong, The structure of compact disjointness preserving operators on continuous functions, Math. Nachr. 282 (2009), 1009-1021. MR2541247 (2010g:47081)

[17] H.H. Schaefer, Topological Vector Spaces, 2nd Edition, Graduate Texts in Mathematics 3, Springer-Verlag, New York, 1999. MR1741419 (2000j:46001)

Department of Mathematics, National University of Singapore, Singapore 119076

E-mail address: matlhh@nus.edu.sg

Department of Mathematics, National Central University, Chungli 32054, Taiwan, Republic of China

E-mail address: wangys@mx.math.ncu.edu.tw

Current address: Department of Mathematical and Statistical Sciences, University of Alberta, Edmonton, Canada

E-mail address: yashu@ualberta.ca 